\title{
Properties of the Margrabe Best-of-two strategy to tactical asset allocation ${ }^{\text {in }}$
}

\author{
David Ardia ${ }^{\mathrm{a}}$, Kris Boudt ${ }^{\mathrm{b}, \mathrm{c}, \mathrm{d}}$, Stefan Hartmann ${ }^{\mathrm{e}}$, Giang Nguyen ${ }^{\mathrm{b}, \mathrm{d}, *}$ \\ a Institute of Financial Analysis, University of Neuchâtel, Switzerland \\ ${ }^{\mathrm{b}}$ Solvay Business School, Vrije Universiteit Brussel, Belgium \\ ${ }^{\mathrm{c}}$ School of Business and Economics, Vrije Universiteit Amsterdam, Netherlands \\ ${ }^{\mathrm{d}}$ Quantitative Strategies, Finvex, Belgium \\ ${ }^{\mathrm{e}}$ ARC Responsible Investment, France
}

\section{A R T I C L E I N F O}

\section{Keywords:}

Best-of-two

Bond-equity

Margrabe

Tactical asset allocation

Upside potential

Downside protection

\begin{abstract}
A B S T R A C T
The Margrabe Best-of-two (MBo2) strategy is a rule-based dynamic investment solution for the twoasset allocation problem. Its typical implementation involves yearly rebalancing the portfolio weights to 50-50 between a low-risk and high-risk asset. It uses intra-year weight adjustments to chase the momentum of the best performing asset by replicating the Margrabe formula for the value of a European option to exchange an asset for another asset at year-end. In practice, this means that the Margrabe portfolio allocation benefits from the upside potential of the high-risk asset and the downside protection from the low-risk asset. The MBo2 allocation depends on the assets' prices, their return volatilities, and correlation, as well as the remaining time until year-end. In this paper, we derive analytical formulae and use simulations to provide insights into the sensitivity of the strategy's weights and performance to these input parameters. We also report the results of an extensive out-ofsample evaluation for the MBo2 strategy applied to the bond-equity, real estate-equity, and world equity-emerging market equity portfolio allocation problems.
\end{abstract}

\section{Introduction}

Bond-equity, equity-gold, domestic equity-emerging markets equity; these are all important two-asset allocation problems. Ideally, the tactical asset allocation is such that the portfolio performs nearly as well as what ex post turns out to be the best-performing asset. ${ }^{1}$ This objective is inherent in a tactical asset allocation that uses the Margrabe Best-of-two (MBo2) strategy. Commercial examples include the NYUSDA index (see, e.g., Kula, Raab, \& Stahn, 2017) and the Metzler Best-of-Germany index, among others. Their investment rule consists of dynamically allocating the portfolio between an equity index and a bond index. The index uses monthly rebalancing. At the end of the year, the portfolio makes a reversal trade by setting the weight allocation to an equal 50-50 investment in the high- and low-risk assets. Within the year, a momentum strategy is pursued by setting weights such that they replicate the value of the Margrabe option to exchange the low-risk asset against the high-risk asset. The initial 50-50 allocation reflects the large uncertainty regarding which asset will outperform on the relatively short one-year horizon.

The Margrabe Best-of-two (MBo2) approach to chase intra-year momentum is an analytically elegant way to combine relative cumulative performance since previous year-end with the predicted probability distribution of the coming end-of-year relative performance. The latter takes also the volatility and correlation of the asset returns into account. An unresolved question is to understand how the drivers of the Margrabe option value interact in determining the MBo2 portfolio

\footnotetext{
Financial support from the Doctiris program of Innoviris is gratefully acknowledged. We are also grateful to UPS Europe and Julie Verstraeten for their support, to

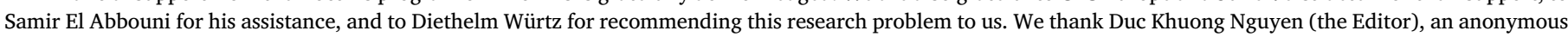

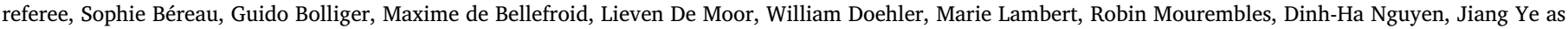

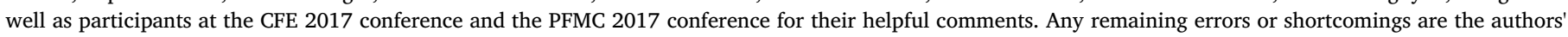
responsibility.

*Corresponding author at: Solvay Business School, Vrije Universiteit Brussel, Belgium.

E-mail addresses: david.ardia@unine.ch (D. Ardia), kris.boudt@vub.be (K. Boudt), stefan.hartmann@arc-ri.com (S. Hartmann), ha.giang.nguyen@vub.be (G. Nguyen).

${ }^{1}$ Examples of other popular two-asset allocation methods include constant-mix, constant proportion portfolio insurance (Black \& Perold, 1992), and core-satellite investing (Amenc, Malaise, \& Martellini, 2004), among others.
} 
weights and performance. We investigate this for both backwardlooking variables, like the relative price of the high versus low-risk asset, and forward-looking parameters, like the high- and low-risk assets' return volatilities and correlation. ${ }^{2}$ We derive explicit formulae for the marginal impact of the input parameters on the portfolio weights, and use numerical experiments to evaluate the impact in case of large changes in the parameters. We then apply historical simulation to investigate the drivers of the performance of the MBo2 strategy over the one- and five-year horizons. In particular, we show that the relative price of the high-risk asset over the low-risk asset and the volatility of the high-risk asset's return are the primary drivers of the portfolio composition and portfolio performance of the MBo2 strategy. The correlation between two assets' returns and the volatility of the low-risk asset's return are found to be less influential. We document the upside potential and downside protection properties of the strategy using realworld and block-bootstrap simulated data.

Our study of the properties of the MBo2 strategy is connected to the large literature that studies the dynamic allocation between two assets. Perold and Sharpe (1995) compare the constant mix and portfolio insurance strategies with the buy-and-hold investment. Cesari and Cremonini (2003) and Ardia, Boudt, and Wauters (2016) provide a comprehensive review of constant-proportion portfolio strategies and the use of simulation techniques to evaluate their performance. Boudt, Darras, Nguyen, and Peeters (2016) compare the use of regimeswitching models with simple trend-following strategies for tactical asset allocation. Kaminski and Lo (2014) study the performance of stoploss policies. Sullivan, Timmermann, and White (1999) highlight the potential issues of data snooping when analyzing the performance of market timing strategies. The use of core-satellite investing is discussed in Welch (2008). Other studies that investigate the impact of mean, variance, and correlation parameters in a more general portfolio setting include Stoyanov, Rachev, and Fabozzi (2013) and Ardia, Bolliger, Boudt, and Gagnon-Fleury (2017). Finally, we refer the reader to Kolm, Tütüncü, and Fabozzi (2014) for a recent review on portfolio optimization.

The paper is organized as follows. We first present the MBo2 strategy in Section 2. Section 3 studies the sensitivity of the MBo2 allocation to the input parameters. The simulation study of determinants of the MBo2 strategy's performance is presented in Section 4. Section 5 concludes. Proofs of the various derivations are presented in the Appendices.

\section{The MBo2 strategy}

\subsection{Definition}

We consider the problem of constructing a portfolio invested in a relatively high-risk asset (such as an equity or a portfolio of equities) and a relatively low-risk asset (such as a government bond or a portfolio of bonds). These assets are denoted by $A$ and $B$, respectively. Their prices at month-end $t$ are denoted by $P_{A, t}$ and $P_{B, t}$.

The Margrabe allocation strategy involves investment decisions at two frequencies. At a low frequency (typically yearly), it sets the weights of the two assets to $50 \%$. At a higher frequency (typically monthly), the portfolio is rebalanced to replicate the value of the Margrabe option to exchange an asset for another asset. Henceforth, we call the low-frequency rebalancing dates as base dates. Without loss of generality, we assume to have only two base dates, namely $t_{0}$ and $t_{1}$, and suppose that the high-frequency rebalancing dates are denoted by $t$, with $t_{0}<t<t_{1}$.

The European option to exchange an asset for another at a maturity

\footnotetext{
${ }^{2}$ In this paper, we use the logarithmic returns of assets' prices to calculate assets' return volatilities and correlation. For portfolio performance, the simple returns of assets' prices are applied.
}

date is computed by Margrabe (1978) under the standard Black-Scholes' assumptions. Before formally defining those weights, we need some more notation. We denote by $T$ the initial investment horizon of the option, defined as the period between the two base dates, $t_{0}$ and $t_{1}$ $\left(t_{1}=t_{0}+T\right)$. We denote by $\Phi(\cdot)$ the standard normal cumulative distribution function.

Consider now the parameters used to set the portfolio weights at time $t$. Let $\tau_{t} \equiv T-t$ be the time to maturity of the option. In order to apply the Margrabe pricing formula, we assume that, for each $t$, the predicted $h$-period ahead volatility and correlation of the monthly returns between $t$ and $t_{1}$ are the same for $h \leq \tau_{t}$. They may however change when $t$ changes. Denote by $\sigma_{A, t}$ and $\sigma_{B, t}$ the annualized volatility of asset $A$ 's and $B$ 's returns. $\rho_{A, B, t}$ is the correlation between asset $A$ 's and $B$ 's returns. We use $\sigma_{A-B, t} \equiv \sqrt{\sigma_{A, t}^{2}+\sigma_{B, t}^{2}-2 \sigma_{A, t} \sigma_{B, t} \rho_{A, B, t}}$ to denote the volatility of the logarithmic return of the relative price.

Finally, we denote by $P_{A, t \mid t_{0}} \equiv P_{A, t} / P_{A, t_{0}}$ and $P_{B, t \mid t_{0}} \equiv P_{B, t} / P_{B, t_{0}}$ their cumulative performance since the reset date $t_{0}$, respectively. We define the relative price of asset $A$ versus asset $B$ at time $t$ as the relative cumulative performance since the reset date $t_{0}$ :

$P_{A / B, t \mid t_{0}} \equiv \frac{P_{A, t \mid t_{0}}}{P_{B, t \mid t_{0}}}$

Margrabe (1978) shows that, under the assumptions of Black and Scholes (1973), the price of the option to exchange asset $B$ for asset $A$ at time $t$ is then given by:

$C_{t}^{B \rightarrow A} \equiv P_{A, t \mid t_{0}} \Phi\left(d_{1, t}\right)-P_{B, t \mid t_{0}} \Phi\left(d_{2, t}\right) \quad$,

where:

$d_{1, t} \equiv \frac{\ln \left(P_{A / B, t \mid t_{0}}\right)+\frac{1}{2} \sigma_{A-B, t}^{2} \tau_{t}}{\sigma_{A-B, t} \sqrt{\tau_{t}}} \quad$ and $\quad d_{2, t} \equiv d_{1, t}-\sigma_{A-B, t} \sqrt{\tau_{t}}$.

Note that the price is an increasing function of the relative price, $P_{A / B, t \mid t_{0}}$, the volatility of the logarithmic return of the relative price, $\sigma_{A-B, t}$, and the time until the maturity of the option, $\tau_{t}{ }^{3}$

The value of the MBo2 replicating portfolio is the sum of the value of asset $B$ and the option to exchange asset $B$ for asset $A$. It is given by:

$P_{\mathrm{MBo} 2, t}=P_{A, t \mid t_{0}} \Phi\left(d_{1, t}\right)+P_{B, t \mid t_{0}}\left(1-\Phi\left(d_{2, t}\right)\right)$.

In Appendix $\mathrm{A}$, we show that this is equivalent to computing $P_{\mathrm{MBo}, t}$ as the sum of the value of asset $A$ and the option to exchange asset $A$ for asset $B$, and thus that $P_{B, t \mid t_{0}}+C_{t}^{B \rightarrow A}=P_{A, t \mid t_{0}}+C_{t}^{A \rightarrow B}$.

From Eq. (2), we obtain directly the portfolio weight that replicates the value of the $\mathrm{MBo} 2$ portfolio, namely:

$w_{A, t} \equiv \frac{P_{A, t \mid t_{0}} \Phi\left(d_{1, t}\right)}{P_{\mathrm{MBo} 2, t}} \quad$ and $\quad w_{B, t} \equiv \frac{P_{B, t \mid t_{0}}\left(1-\Phi\left(d_{2, t}\right)\right)}{P_{\mathrm{MBo} 2, t}}$

In Appendix B, we show that, under the standard Black-Scholes' assumptions, it is equivalent to calculate the weight of the high-risk asset (asset $A$ ) in the $\mathrm{MBo} 2$ replicating portfolio as:

$$
\begin{aligned}
w_{A, t} & =\frac{P_{A / B, t \mid t_{0}} \Phi\left(d_{1, t}\right)}{P_{A / B, t \mid t_{0}} \Phi\left(d_{1, t}\right)+\left(1-\Phi\left(d_{2, t}\right)\right)} \\
& =\frac{\mathbb{E}\left[P_{A / B, T \mid t} \mid P_{A / B, T \mid t}>1\right]}{\mathbb{E}\left[P_{A / B, T \mid t} \mid P_{A / B, T \mid t}>1\right]+\mathbb{P}\left[P_{A / B, T \mid t}<1\right]} .
\end{aligned}
$$

The high-risk asset weight in the replicating portfolio then equals the ratio between its expected relative price, when it exceeds one, and the sum of that value and the probability that the relative price ends lower than one at maturity.

Since the portfolio is fully invested, the weight of the low-risk asset (asset $B$ ) in the portfolio is $w_{B, t} \equiv 1-w_{A, t}$. By construction, all weights

\footnotetext{
${ }^{3}$ The latter two become clear by considering the special case where $P_{A, t \mid t_{0}}=P_{B, t \mid t_{0}}$. Then the option price equals $P_{A, t \mid t_{0}}\left(2 \Phi\left(\frac{1}{2} \sigma_{A-B, t} \sqrt{\tau_{t}}\right)-1\right)$, which is increasing in $\sigma_{A-B, t}$ and $\tau_{t}$.
} 
are in the range of $[0,1]$. In Appendix $C$, we show that, in the special case where $P_{A, t \mid t_{0}}=P_{B, t \mid t_{0}}$ (and thus $P_{A / B, t \mid t_{0}}=1$ ), the weight of the highrisk asset is 0.5 irrespective of the values for the other parameters. On each of the low-frequency base dates, $t_{0}$ and $t_{1}$, we thus have $w_{A, t}=0.5$. Between the rebalancing dates, the weights automatically adjust in function of the relative performance of the assets $A$ and $B$.

\subsection{Illustration of the $\mathrm{MBo} 2$ strategy}

The MBo2 strategy leads to a tactical allocation that, when compared to buy-and-hold investments in one of the underlying assets, has the advantage of benefitting from the upside potential of the high-risk asset and from the downside protection of the low-risk asset. We illustrate this here in the case of high and low-risk assets chosen for investment in the US, Germany, and international markets. The equity-bond investments in the US and Germany are inspired by the NYUSDA index and Metzler Best-of-Germany tactical asset allocation solutions. The investment problems that we study are fourfold. Our main analysis is in terms of the allocation problem between US equities (S\&P500 total return index, in USD) and US bonds (Barclays US Treasury 7-10 year total return index, in USD). In addition, we study the problem of allocating between US real estate (NAREIT total return index, in USD) and US equities (S\&P500 total return index, in USD), between MSCI Emerging market equity (EM total return index, in USD) and MSCI World equity (DE total return index, in USD), and between German equities (DAX total return index, in EUR) and German bonds (Germany Treasury 7-10 year total return index, in EUR).

For each of these applications, we compare the performance of the MBo2 with two traditional tactical allocation methods: (i) buy-and-hold investments in one underlying, and (ii) monthly rebalanced constantmix portfolios. ${ }^{4}$ We consider the performance over the period ranging from January 1992 to March 2017 (303 monthly observations). ${ }^{5}$

For this practical illustration, we have more than one reset-date. We denote these dates by $t_{0} \in\left\{t_{0,1}, t_{0,2} \ldots t_{0, K}\right\}$ where the distance between any two consecutive dates is exactly equal to the investment horizon of the option ( $T$ ). In the base model, the base date is the last trading day of each year. On these base dates, the relative price is set at one and the weights on equity and bond are set to $50-50$. Volatilities and correlation of two assets' returns are estimated using a rolling window of three years of monthly returns (36 observations). The estimated values on a base date $t_{0, k}$ are kept constant during the investment horizon of the option which is assumed to be 12 months $(T=12)$ until the next base date $t_{0, k+1}$. The portfolio weights are rebalanced on a monthly basis with the update of the relative price and the time to maturity of the option. The out-of-sample window is from February 1995 to March 2017.

Fig. 1 displays the cumulative performance of the buy-and-hold strategies together with the MBo2 strategy for the equity-bond investment in the US (top-left panel), NAREIT-equity investment in the US market (top-right panel), equity emerging-developed markets (bottom-left panel), and the equity-bond investment in the German market (bottom-right panel). We see that the cumulative values of the buy-and-hold strategy on the low-risk asset are more stable than those invested in the high-risk asset. This comes at the price of a lower performance. Regarding the MBo2 strategy, we see that volatility is between the values of the buy-and-hold strategy on the high-risk asset and

\footnotetext{
${ }^{4}$ The market risk-free rate in the US is the T-bill one-month yields collected from the K. French data library: http://mba.tuck.dartmouth.edu/pages/ faculty/ken.french/data_library.html. The risk-free rate in Germany is the three-month interbank yields, available at the website of the Federal Reserve Bank of St. Louis: https://fred.stlouisfed.org/.

${ }^{5}$ The publicly available series of return performance for NYUSDA index and Metzler Best-of-Germany are available from January 2000 and June 2000 onward, respectively.
}

low-risk asset, respectively. It outperforms in terms of a higher end-ofperiod cumulative value and is more stable, as it avoids large losses in the bearish periods of 2000-2002 (the dot-com crisis) and 2007-2008 (the global financial crisis), and participated in the upside potential of high-risk assets in the bullish period.

In order to gauge the risk-return properties of these investment solutions, we compare in Table 1 the out-of-sample performance statistics of the MBo2 strategy with those of the buy-and-hold strategy on each asset, and the constant-mix strategies of 50/50 equal-weighting and $60 / 40$ weighting.

In Panel A of Table 1, we find that, for the US market, the MBo2 strategy has the highest annualized return (10.69\%) and the highest Sharpe ratio (0.77) over the out-of-sample evaluation period February 1995-March 2017. Its volatility is lower than the buy-and-hold strategy on the high-risk asset and the constant-mix 60/40 strategy $(8.52 \%$ versus $14.82 \%$ and $8.72 \%$ ). The buy-and-hold strategy on the bond index has the lowest values of volatility and drawdown $(6.26 \%$ and $7.37 \%$, respectively). The lower risk comes at the cost of a lower return (6.24\%). The reverse applies to the buy-and-hold strategy on the equity index. The equally-weighted or constant-mix 60/40 strategies generate returns, volatilities, and drawdowns in the range of the buy-and-hold strategy on equities and bonds. It is interesting to note that the worst drawdown of the MBo2 strategy (14.3\%) was in August 1998 when the market crashed by $14.6 \%$ in a month. Meanwhile, during the financial crisis of 2008, the MBo2 strategy only suffered from a drawdown of $10.0 \%$ compared with $51 \%$ for the buy-and-hold strategy on the equity. This illustrates the downside risk protection offered by the MBo2 strategy compared to buy-and-hold investment in the high-risk asset.

As can be expected, the monthly rebalancing to replicate the value of the Best-of-two portfolio and the yearly rebalancing to the 50-50 constant mix leads to a relatively high annualized turnover of $62.53 \%$. To show that the outperformance in terms of higher Sharpe ratio is also to be expected in terms of net returns (after transaction costs), we report in the last column of Table 1 the so-called break-even transaction costs (BETC) of the MBo2 versus the four alternative investment strategies. The BETC is defined as the fee (expressed in cents per dollar traded) that the MBo2 strategy can charge such that it has, in net returns, an equal Sharpe ratio with the alternative strategy. As can be seen in Table 1, it varies between 0.86 and 1.78 cents per dollar traded. We can thus conclude that the outperformance of the MBo2 strategy in terms of Sharpe ratio is high enough to be robust to realistic values of transaction cost.

Similar results are observed for the NAREIT-equity investment, the equity emerging-developed markets, and the German equity-bond investment, where we find that the MBo2 strategy yields the highest return, and a lower volatility and drawdown than the buy-and-hold strategy on the high-risk asset.

\section{Drivers of the weight allocation of the MBo2 strategy}

From the weight definition in Eq. (3), it follows that the MBo2 weights are a non-linear function of various parameters. In this section, we shed more light on the sensitivity of the weights to those parameters. We first compute the partial derivatives of the weight of the high-risk asset $A$ with respect to each of the input parameters. ${ }^{6}$ These derivatives show the direction and the magnitude of the effect of an infinitesimal change in the input parameters. We then use a numerical evaluation to quantify the effects of larger changes in the magnitude of the parameters.

\subsection{High-risk asset weight partial sensitivity to the input parameters}

A crucial feature of the momentum interpretation of the MBo2

${ }^{6}$ The derivatives for the low-risk asset $B$ follow directly from $w_{B, t} \equiv 1-w_{A, t}$. 

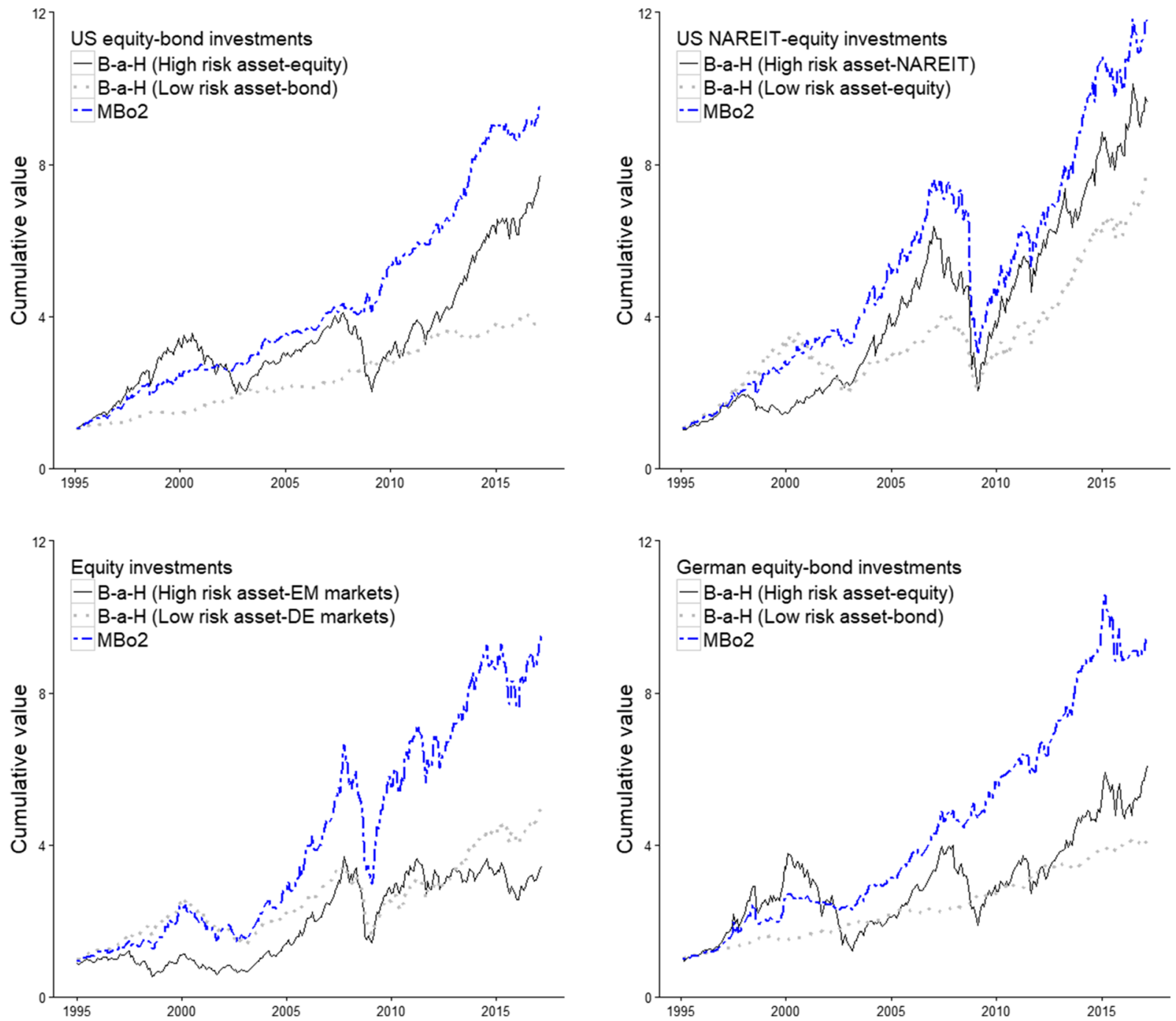

Fig. 1. Cumulative values of strategies on two-asset allocation. The figure displays the out-of-sample cumulative values of buy-and-hold strategies in the high-risk asset (the full black line), the low-risk asset (the gray dotted line), and the MBo2 strategy (the blue dashed line) over the period February 1995 to March 2017 . Topleft chart shows the cumulative value of $\$ 1$ invested in equity and bond in the U.S market. Top-left chart shows the cumulative value of $\$ 1$ invested in real estate and equity in the U.S market. Bottom-left chart shows the cumulative value of $\$ 1$ invested in international equity markets. Bottom-right chart shows the cumulative value of $€ 1$ invested in equity and bond in the German market. See Section 2.2 for details. (For interpretation of the references to color in this figure legend, the reader is referred to the web version of this article.)

weight allocation is that the weight of the high-risk asset is an increasing function of the relative price of the high-risk asset regarding the price of the low-risk asset. It implies that the partial derivative of the high-risk asset weight with respect to its relative price must be positive. We prove this in Appendix D, where we obtain the following expression:

$$
\begin{aligned}
\frac{\partial w_{A, t}}{\partial P_{A / B, t \mid t_{0}}}= & \frac{1}{\left[P_{A / B, t \mid t_{0}} \Phi\left(d_{1, t}\right)+(\underbrace{}_{>0}\left(1-\Phi\left(d_{2, t}\right)\right)]^{2}\right.} \\
& \times\left\{\left(1-\Phi\left(d_{2, t}\right)\right) \Phi\left(d_{1, t}\right)+\frac{1}{\sigma_{A-B, t} \underbrace{\sqrt{\tau_{t}}}_{\geq 0}}\left[\left(1-\Phi\left(d_{2, t}\right)\right) \phi\left(d_{1, t}\right)+\phi\left(d_{2, t}\right)\right]\right\}
\end{aligned}
$$

In Appendices E-H, we show that the partial derivatives of the weight of the high-risk asset with respect to the other parameters (return's volatility, correlation, and the time to maturity of the Margrabe option) have a common structure, namely:

$$
\begin{aligned}
& \frac{\partial w_{A, t}}{\partial \sigma_{A, t}}=\kappa_{t} \times(\sigma_{A, t}-\underbrace{\sigma_{B, t} \rho_{A, B, t}}_{>0}) \\
& \frac{\partial w_{A, t}}{\partial \sigma_{B, t}}=\kappa_{t} \times\left(\sigma_{B, t}-\sigma_{A, t} \rho_{A, B, t}\right) \\
& \frac{\partial w_{A, t}}{\partial \rho_{A, B, t}}=(-1) \times \kappa_{t} \times \sigma_{A, t} \sigma_{B, t} \\
& \frac{\partial w_{A, t}}{\partial \tau_{t}}=\kappa_{t} \times \frac{1}{2} \sigma_{A-B, t},
\end{aligned}
$$

where we use that, by definition of the high-risk asset, $\sigma_{A, t}>\sigma_{B, t}$ and thus $\sigma_{A, t}-\sigma_{B, t} \rho_{A, B, t}>0$. The common parameter $\kappa_{t}$ is given by: 
Table 1

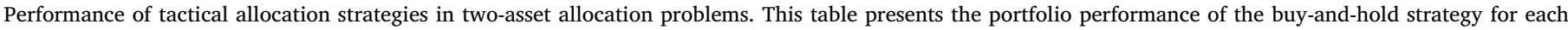

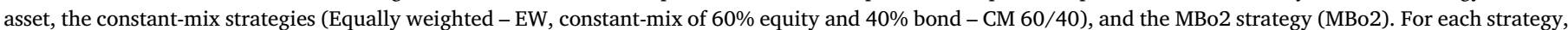

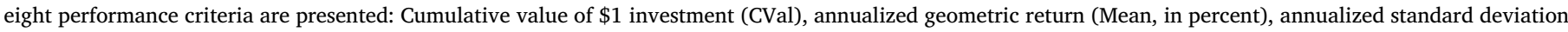

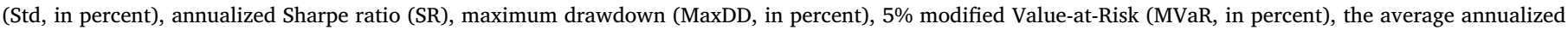

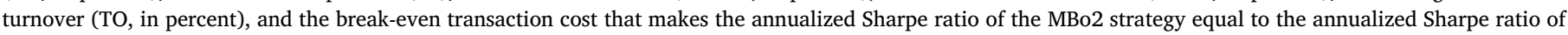

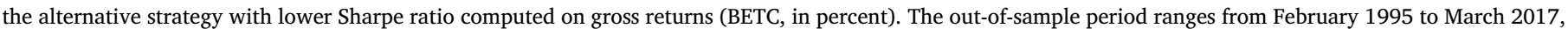
for a total of 266 monthly observations. See Section 2.2 for details.

\begin{tabular}{|c|c|c|c|c|c|c|c|c|}
\hline Strategies & CVal & Mean & Std & SR & MaxDD & MVaR & TO & BETC \\
\hline \multicolumn{9}{|c|}{ Panel A: tactical allocation strategies on equity-bond investments in the US market } \\
\hline Equity & 7.68 & 9.64 & 14.82 & 0.37 & 50.95 & 6.85 & - & 1.78 \\
\hline Bond & 3.82 & 6.24 & 6.26 & 0.36 & 7.37 & 2.37 & - & 1.43 \\
\hline EW & 5.89 & 8.33 & 7.41 & 0.57 & 23.28 & 3.08 & 6.14 & 0.86 \\
\hline CM 60/40 & 6.30 & 8.66 & 8.72 & 0.52 & 29.69 & 3.75 & 5.91 & 1.14 \\
\hline MBo2 & 9.51 & 10.69 & 8.52 & 0.77 & 14.30 & 3.35 & 62.53 & - \\
\hline \multicolumn{9}{|c|}{ Panel B: tactical allocation strategies on real estate-equity investments in the US market } \\
\hline NAREIT & 9.64 & 10.76 & 18.85 & 0.35 & 67.89 & 8.42 & - & 3.56 \\
\hline Equity & 7.68 & 9.64 & 14.82 & 0.37 & 50.95 & 6.85 & - & 3.32 \\
\hline EW & 9.25 & 10.56 & 14.94 & 0.43 & 58.2 & 6.89 & 5.72 & 3.02 \\
\hline CM $60 / 40$ & 9.44 & 10.66 & 15.47 & 0.42 & 59.96 & 7.09 & 5.50 & 3.09 \\
\hline MBo2 & 11.76 & 11.76 & 15.56 & 0.49 & 60.61 & 7.62 & 56.84 & - \\
\hline \multicolumn{9}{|c|}{ Panel C: tactical allocation strategies on international equity investments } \\
\hline EM equity & 3.41 & 5.67 & 22.98 & 0.10 & 61.44 & 11.18 & - & 3.68 \\
\hline DE equity & 4.94 & 7.44 & 14.93 & 0.24 & 53.65 & 7.11 & - & 2.52 \\
\hline EW & 4.34 & 6.82 & 18.09 & 0.18 & 57.52 & 8.76 & 4.96 & 3.07 \\
\hline $\mathrm{CM} 60 / 40$ & 4.17 & 6.63 & 18.97 & 0.16 & 58.30 & 9.19 & 4.78 & 3.19 \\
\hline MBo2 & 9.50 & 10.65 & 18.18 & 0.38 & 55.44 & 7.97 & 58.12 & - \\
\hline \multicolumn{9}{|c|}{ Panel D: tactical allocation strategies on equity-bond investments in the German market } \\
\hline Equity & 6.08 & 8.48 & 21.49 & 0.23 & 68.29 & 9.98 & - & 2.50 \\
\hline Bond & 4.06 & 6.52 & 4.79 & 0.64 & 7.61 & 1.75 & - & - \\
\hline EW & 5.79 & 8.24 & 10.38 & 0.45 & 33.56 & 4.45 & 8.31 & 1.23 \\
\hline CM $60 / 40$ & 6.00 & 8.42 & 12.53 & 0.39 & 42.20 & 5.52 & 8.03 & 1.67 \\
\hline MBo2 & 9.48 & 10.68 & 12.00 & 0.59 & 20.53 & 4.49 & 57.34 & - \\
\hline
\end{tabular}

$$
\begin{aligned}
\kappa_{t} \equiv & \frac{P_{A / B, t \mid t_{0}} \sqrt{\tau_{t}}}{\left[P_{A / B, t \mid t_{0}} \Phi\left(d_{1, t}\right)+\left(1-\Phi\left(d_{2, t}\right)\right)\right]^{2}} \\
\times & {\left[\left(1-\Phi\left(d_{2, t}\right)\right) \phi\left(d_{1, t}\right)\left(1-\frac{\ln \left(P_{A / B, t \mid t_{0}}\right)}{\sigma_{A-B, t}^{2} \tau_{t}}\right)\right.} \\
& \left.-\Phi\left(d_{1, t}\right) \phi\left(d_{2, t}\right)\left(\frac{\ln \left(P_{A / B, t \mid t_{0}}\right)}{\sigma_{A-B, t}^{2} \tau_{t}}+1\right)\right] .
\end{aligned}
$$

A sufficient condition for $\kappa_{t}$ to be negative is that $\ln \left(P_{A / B, t \mid t_{0}}\right) \geq \sigma_{A-B, t}^{2} \tau_{t}$, which means that $P_{A / B, t \mid t_{0}} \geq \exp \left(\sigma_{A-B, t}^{2} \tau_{t}\right)$. When the high-risk asset outperforms the low-risk asset, we thus have that an increase of the volatility of the high-risk leads to a decrease of the high-risk asset's weight in the MBo2 replicating portfolio. Such directional impact is the same as the time to the maturity and the volatility of the low-risk asset's return (under the condition that $\rho_{A, B, t}<\sigma_{B, t} / \sigma_{A, t}$ ). The impact is of opposite sign as compared to the impact of the correlation of two assets' returns.

\subsection{Numerical study of the sensitivity of the high-risk asset weight to the input parameters}

We now illustrate the sensitivity of the high-risk asset weight to larger (non-infinitesimal) changes in the parameters in a stylized setup and quantify the magnitude of the impact. We first describe the calibration of the parameters and then discuss the results reported in Table 2.

Throughout the table, we assume, as the reference scenario, that the relative price of asset $A$ over $B$ is $1.1\left(P_{A / B, t \mid t_{0}}=1.1\right)$. The alternative values that we consider are 0.9 and 1 . For the volatility of the high-risk and low-risk asset return series, we consider the sets $\{5 \%, 25 \%, 45 \%$ \} and $\{4 \%, 6 \%, 8 \%$, with $25 \%$ and $6 \%$ as our reference values. The reference correlation is -0.2 . The alternative values considered are -0.7 and 0.3 . The initial investment horizon of the MBo2 investment strategy is 12 months and the remaining time to maturity $\tau_{t}$ is either 3,7 or 11 months. The reference value is 7 months. In terms of the key parameters of Eqs. (5)-(8), we have that the setting of $\left(P_{A / B, t \mid t_{0}}, \sigma_{A, t}, \sigma_{B, t}, \rho_{A, B, t}, \tau_{t}\right)=(1.1,0.25,0.06,-0.2,7 / 12) \quad$ leads to $\kappa_{t}=-0.16$ and $\sigma_{B, t}-\rho_{A, B, t} \sigma_{A, t}=0.11$.

Panel A of Table 2 shows how the high-risk asset weight changes when modifying the relative performance of the high-risk asset compared to the low-risk asset, while keeping all other variables constant. In the reference scenario, the higher risk of asset $A$ is rewarded in terms of a $10 \%$ higher relative realized performance. Of course, in reality, the relative performance can be even higher, but also below one. The MBo2 strategy adapts itself to it. In fact, since the partial derivative of the weight of the high-risk asset with respect to the relative price is positive (as Eq. (4)), the weight on the high-risk asset increases when the relative price increases, ceteris paribus. This can be seen in the column of the numbers in bold in Panel A of Table 2. The weight varies from $0.51 \%$ to $95.59 \%$ for a range of relative prices from 0.6 to 1.4 , respectively. For all columns, we see that the weight of the high-risk asset increases when the relative price increases.

In Panel A of Table 2, we also investigate the sensitivity of the highrisk asset weight to the volatility of the underlying asset returns. The weights in the MBo2 strategy are based on the projected distribution of end-of-year relative performance, which depends on the volatility of the high-risk and low-risk assets' returns. Since volatility is time-varying, it is important to gauge the impact of changes in volatility on the portfolio weights. The second block of Panel A shows that the weights are indeed very sensitive to the volatility of the high-risk asset's return. ${ }^{7}$ At a relative price of 1.1, the weights of the high-risk asset are $93.05 \%$, $68.68 \%$, and $61.44 \%$ for the volatility of the high-risk asset's return at

\footnotetext{
${ }^{7}$ We do not report the second block in Panel B and Panel C as they are the same as in Panel A.
} 
Table 2

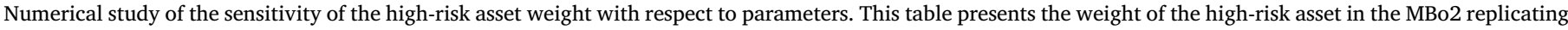

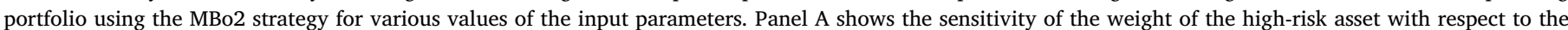

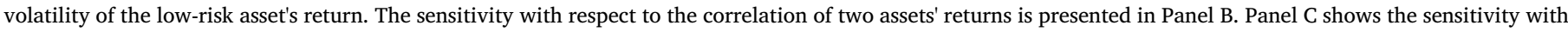

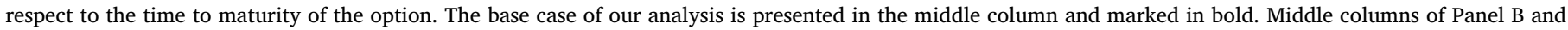
Panel C are skipped as they are the same as those of Panel A. See Section 3 for details.

\begin{tabular}{|c|c|c|c|c|c|c|c|c|c|c|c|c|c|c|c|}
\hline \multirow[b]{2}{*}{$P_{A / B, t \mid t_{0}}$} & \multicolumn{5}{|c|}{$\sigma_{B, t}=4 \%$ and $\sigma_{A, t}=$} & \multicolumn{5}{|c|}{$\sigma_{B, t}=6 \%$ and $\sigma_{A, t}=$} & \multicolumn{5}{|c|}{$\sigma_{B, t}=8 \%$ and $\sigma_{A, t}=$} \\
\hline & $5 \%$ & $15 \%$ & $25 \%$ & $35 \%$ & $45 \%$ & $5 \%$ & $15 \%$ & $25 \%$ & $35 \%$ & $45 \%$ & $5 \%$ & $15 \%$ & $25 \%$ & $35 \%$ & $45 \%$ \\
\hline 0.60 & 0.00 & 0.00 & 0.41 & 2.56 & 5.97 & 0.00 & 0.00 & 0.51 & 2.76 & 6.19 & 0.00 & 0.01 & 0.63 & 3.00 & 6.44 \\
\hline 0.70 & 0.00 & 0.18 & 3.18 & 8.53 & 13.70 & 0.00 & 0.29 & 3.55 & 8.90 & 13.99 & 0.00 & 0.47 & 4.00 & 9.31 & 14.31 \\
\hline 0.80 & 0.00 & 3.32 & 12.17 & 19.46 & 24.60 & 0.03 & 4.12 & 12.81 & 19.86 & 24.86 & 0.20 & 5.11 & 13.54 & 20.31 & 25.14 \\
\hline 0.90 & 2.33 & 19.20 & 29.02 & 34.17 & 37.25 & 5.11 & 20.51 & 29.53 & 34.43 & 37.40 & 8.58 & 21.92 & 30.09 & 34.71 & 37.56 \\
\hline 1.00 & 50.00 & 50.00 & 50.00 & 50.00 & 50.00 & 50.00 & 50.00 & 50.00 & 50.00 & 50.00 & 50.00 & 50.00 & 50.00 & 50.00 & 50.00 \\
\hline 1.10 & 96.41 & 78.45 & 69.15 & 64.39 & 61.57 & 93.05 & 77.19 & 68.68 & 64.16 & 61.44 & 89.20 & 75.84 & 68.15 & 63.90 & 61.29 \\
\hline 1.20 & 99.97 & 93.35 & 83.01 & 75.94 & 71.29 & 99.76 & 92.24 & 82.35 & 75.57 & 71.06 & 99.08 & 90.94 & 81.62 & 75.16 & 70.81 \\
\hline 1.30 & 100.00 & 98.44 & 91.46 & 84.40 & 79.02 & 100.00 & 97.93 & 90.87 & 83.99 & 78.74 & 99.96 & 97.25 & 90.20 & 83.54 & 78.43 \\
\hline 1.40 & 100.00 & 99.71 & 96.01 & 90.21 & 84.91 & 100.00 & 99.54 & 95.59 & 89.83 & 84.62 & 100.00 & 99.29 & 95.09 & 89.40 & 84.30 \\
\hline
\end{tabular}

Panel B: $w_{A, t}$ for various values of the return correlation $\rho_{A, B, t}$

\begin{tabular}{|c|c|c|c|c|c|c|c|c|c|c|c|c|c|c|c|}
\hline \multirow[b]{2}{*}{$P_{A / B, t \mid t_{0}}$} & \multicolumn{5}{|c|}{$\rho_{A, B, t}=-0.7$ and $\sigma_{A, t}=$} & \multicolumn{5}{|c|}{$\rho_{A, B, t}=-0.2$ and $\sigma_{A, t}=$} & \multicolumn{5}{|c|}{$\rho_{A, B, t}=0.3$ and $\sigma_{A, t}=$} \\
\hline & $5 \%$ & $15 \%$ & $25 \%$ & $35 \%$ & $45 \%$ & $5 \%$ & $15 \%$ & $\mathbf{2 5} \%$ & $35 \%$ & $45 \%$ & $5 \%$ & $15 \%$ & $25 \%$ & $35 \%$ & $45 \%$ \\
\hline 0.60 & 0.00 & 0.03 & 0.94 & 3.65 & 7.23 & & & & & & 0.00 & 0.00 & 0.20 & 1.90 & 5.10 \\
\hline 0.70 & 0.00 & 0.75 & 4.94 & 10.39 & 15.28 & & & & & & 0.00 & 0.05 & 2.18 & 7.24 & 12.54 \\
\hline 0.80 & 0.18 & 6.32 & 14.96 & 21.43 & 25.99 & & & & & & 0.00 & 1.93 & 10.23 & 17.97 & 23.53 \\
\hline 0.90 & 8.38 & 23.43 & 31.14 & 35.40 & 38.04 & & & & & & 1.69 & 16.32 & 27.38 & 33.21 & 36.63 \\
\hline 1.00 & 50.00 & 50.00 & 50.00 & 50.00 & 50.00 & & & & & & 50.00 & 50.00 & 50.00 & 50.00 & 50.00 \\
\hline 1.10 & 89.42 & 74.40 & 67.18 & 63.27 & 60.85 & & & & & & 97.26 & 81.27 & 70.68 & 65.27 & 62.13 \\
\hline 1.20 & 99.14 & 89.45 & 80.22 & 74.13 & 70.07 & & & & & & 99.99 & 95.48 & 85.04 & 77.33 & 72.25 \\
\hline 1.30 & 99.97 & 96.37 & 88.86 & 82.37 & 77.51 & & & & & & 100.00 & 99.24 & 93.18 & 85.91 & 80.17 \\
\hline 1.40 & 100.00 & 98.91 & 94.05 & 88.28 & 83.33 & & & & & & 100.00 & 99.91 & 97.16 & 91.57 & 86.09 \\
\hline
\end{tabular}

Panel C: $w_{A, t}$ for various values of the time to maturity of the option $\tau_{t}$

\begin{tabular}{|c|c|c|c|c|c|c|c|c|c|c|c|c|c|c|c|}
\hline \multirow[b]{2}{*}{$P_{A / B, t \mid t_{0}}$} & \multicolumn{5}{|c|}{$\tau_{t}=3$ and $\sigma_{A, t}=$} & \multicolumn{5}{|c|}{$\tau_{t}=7$ and $\sigma_{A, t}=$} & \multicolumn{5}{|c|}{$\tau_{t}=11$ and $\sigma_{A, t}=$} \\
\hline & $5 \%$ & $15 \%$ & $25 \%$ & $35 \%$ & $45 \%$ & $5 \%$ & $15 \%$ & $\mathbf{2 5} \%$ & $35 \%$ & $45 \%$ & $5 \%$ & $15 \%$ & $25 \%$ & $35 \%$ & $45 \%$ \\
\hline 0.60 & 0.00 & 0.00 & 0.01 & 0.21 & 1.13 & & & & & & 0.00 & 0.08 & 1.89 & 5.97 & 10.46 \\
\hline 0.70 & 0.00 & 0.00 & 0.34 & 2.23 & 5.47 & & & & & & 0.00 & 1.32 & 7.22 & 13.70 & 18.90 \\
\hline 0.80 & 0.00 & 0.44 & 4.42 & 10.33 & 15.70 & & & & & & 0.29 & 8.12 & 17.95 & 24.60 & 29.00 \\
\hline 0.90 & 0.65 & 10.67 & 20.96 & 27.47 & 31.67 & & & & & & 9.52 & 25.39 & 33.20 & 37.25 & 39.67 \\
\hline 1.00 & 50.00 & 50.00 & 50.00 & 50.00 & 50.00 & & & & & & 50.00 & 50.00 & 50.00 & 50.00 & 50.00 \\
\hline 1.10 & 98.77 & 86.99 & 76.76 & 70.59 & 66.69 & & & & & & 88.20 & 72.55 & 65.28 & 61.57 & 59.36 \\
\hline 1.20 & 100.00 & 98.41 & 91.84 & 84.93 & 79.49 & & & & & & 98.81 & 87.36 & 77.35 & 71.29 & 67.46 \\
\hline 1.30 & 100.00 & 99.90 & 97.73 & 93.09 & 88.14 & & & & & & 99.94 & 94.95 & 85.93 & 79.01 & 74.22 \\
\hline 1.40 & 100.00 & 100.00 & 99.47 & 97.10 & 93.47 & & & & & & 100.00 & 98.20 & 91.59 & 84.91 & 79.73 \\
\hline
\end{tabular}

$5 \%, 25 \%$, and $45 \%$, respectively. The decrease in weight of the highrisk asset when its volatility increases reflects the decrease in the probability that the relative price at maturity still exceeds one.

Comparing the bold column (the volatility of the high-risk asset's return is at $25 \%$ ), and changing the volatility of the low-risk asset from $4 \%$ (the middle column in the first block) to $8 \%$ (the middle column in the third block), the weight does not change much (e.g., at the relative price level of 1.2 , the weights are $83.01 \%$ and $81.62 \%$ if the volatility of the low-risk asset is at $4 \%$ and $8 \%$, respectively versus $82.35 \%$ of the base case where the volatility of the low-risk asset is 6\%.).

The high-risk asset weight also depends on the correlation between the asset returns. The reference correlation between the high-risk and low-risk asset return is -0.2 , indicating the diversification potential of combining the high- and low-risk asset in the portfolio. In the case of a flight to safety, we expect the correlation to become more negative, while in some market regimes the correlation can as well become positive. These changes in correlation affect the outcome in the MBo2 portfolio weights. From Eq. (7), the effect of the correlation is opposite to the sign of $\kappa_{t}$. As $\kappa_{t}$ in our reference case is negative, an increase in correlation leads to an increase in the weight. This effect is however weak. In fact, as can be seen in Panel B of Table 2, the correlation has less impact on the weight of the high-risk asset in the MBo2 replicating portfolio than the impact of volatility. Particularly, when the relative price is 1.1 , ceteris paribus, the weight of the high-risk asset is $67.18 \%$, $68.68 \%$, and $70.68 \%$ for correlation values of $-0.7,-0.2$, and 0.3 , respectively.

In Panel C of Table 2, we analyze the sensitivity of the high-risk asset weight to the time to maturity. The $\tau_{t}$ parameter in the MBo2 formula deterministically changes with time. According to Eq. (8), the directional impact of $\tau_{t}$ on the high-risk asset weight positively relates to $\kappa_{t}$. In our reference case, $\kappa_{t}$ is negative. When the time to maturity decreases, the high-risk asset weight thus increases. This can be seen in Panel C where the relative price is 1.1, ceteris paribus, the high-risk asset weight increases slightly $(65.28 \%, 68.68 \%$, and $76.76 \%)$ for decreasing values of time to maturity (eleven, seven, and three periods until the maturity). The impact is thus moderate.

Table 2 illustrates the dependence of the high-risk asset weight on input parameters for a small grid of values. In Fig. 3, we plot the highrisk asset weight as a continuous function of the relative price for various values of the other parameters. In Fig. 2 we see that when the 

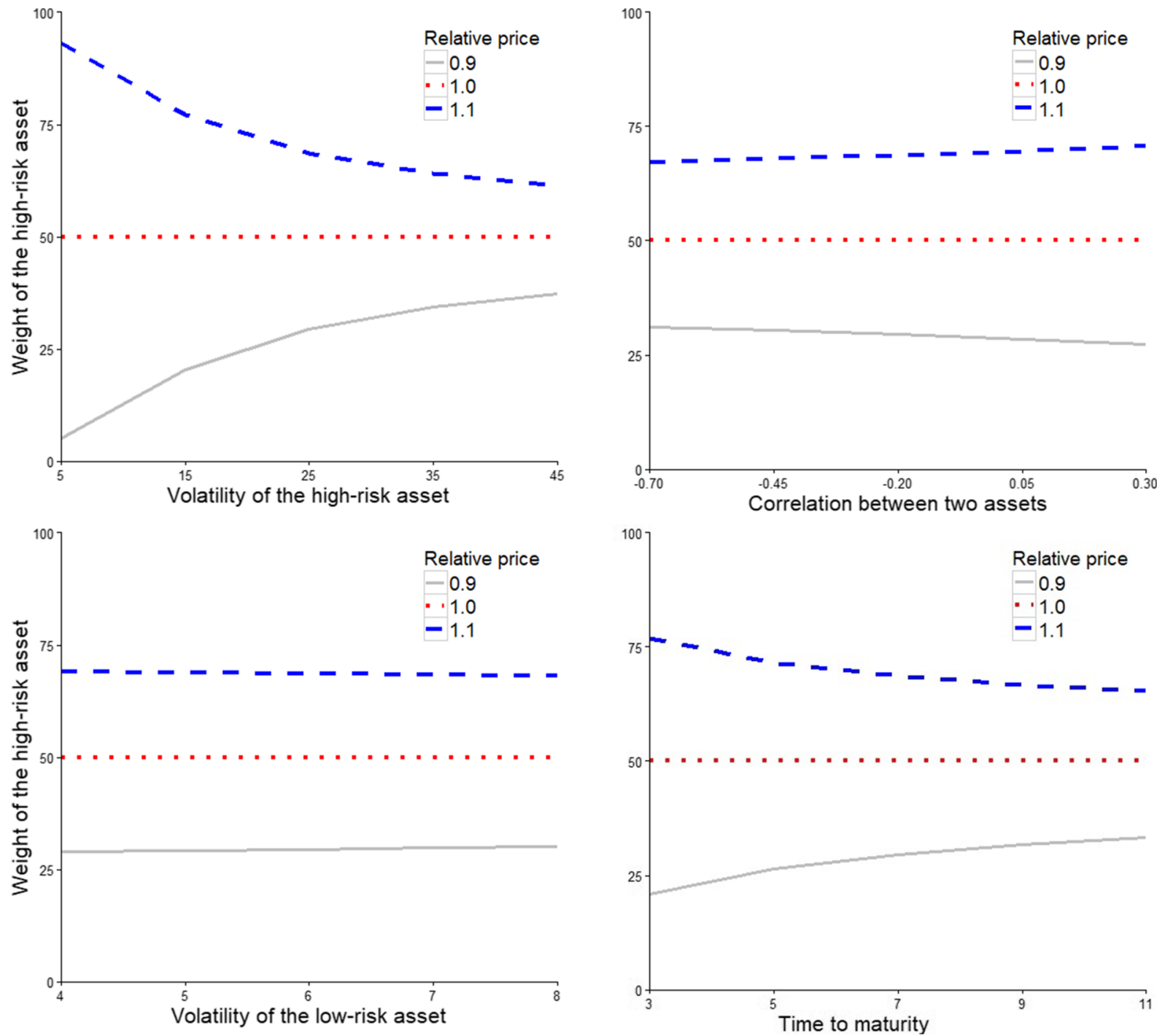

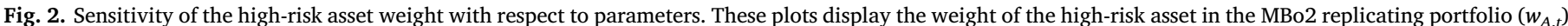

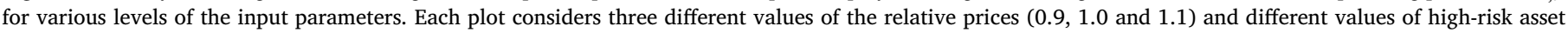
volatility (top left), correlation (top right), the low-risk asset volatility (bottom left), and time to maturity (bottom right). See Table 2 for details.

relative price is at 1.0 , the weight of the high-risk asset is always $50 \%$ regardless of the value of the other parameters. In case of a relative price of 0.9, we find that an increase in volatilities of two assets' returns and the number of periods until maturity lead to an increase of the weight of the high-risk asset. In contrast, an increase of the correlation leads to a slight decrease of the weight. The opposite holds for a relative price of 1.1.

Fig. 3 shows that the weight of the high-risk asset is extremely different in cases of a low and high value of the high-risk 's asset volatility ( $5 \%$ and $45 \%$, respectively). In particular, when the volatility of the high-risk asset's return is $5 \%$, the high-risk asset's weight increases from $5.11 \%$ to $50 \%$ when the relative price increases from 0.9 to 1.0 . Meanwhile, the high-risk asset weight only increases from and $37.40 \%$ to $50 \%$ when the volatility of the high-risk asset's return is $45 \%$. Overall, at the high volatility of the high-risk asset's return (45\%), the high-risk asset weight in the replicating portfolio is almost a linear function of the relative price with a positive slope. This is not the case for the low volatility level of the low-risk asset, where the weight of the high-risk asset does not change.

Regarding the correlation between two assets' returns, one can note that the gap between the two lines of the weight of the high-risk asset is smaller than those of the volatility of the high-risk asset's returns and the time to maturity of the option. The impact on the weight of the high-risk asset is, therefore, less noticeable than those of the volatility and the time to maturity of the option.

The difference between the two extreme values of the time to maturity of the Margrabe option is less obvious than the volatility of the high-risk asset but clearer than the correlation. For all parameters, we see the non-linear and asymmetric relation between the relative price and the weight of the high-risk asset in the $\mathrm{MBo} 2$ replicating portfolio. In addition, when the relative price is either extremely high/low or close to one, the differences are smaller than those in the other cases.

\section{The non-linearity between the performance of the high-risk asset and the MBo2 strategy}

In this section, we study the portfolio performance of the MBo2 strategy for a one-year and a five-year investment horizon using simulated price-paths. We illustrate that the MBo2 is an investment strategy that benefits from the upside potential of the high-risk asset and the downside protection of the low-risk asset. 

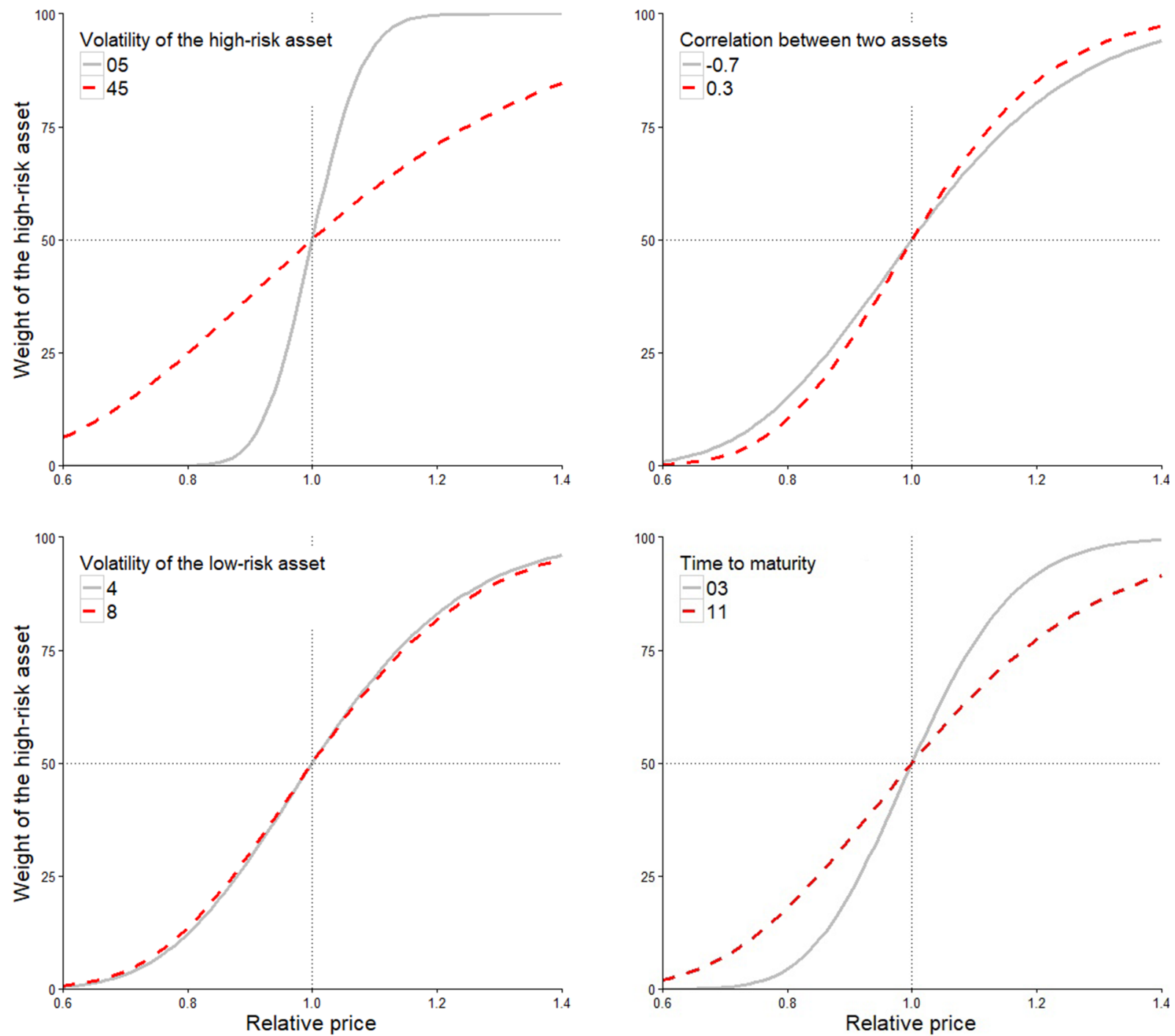

Fig. 3. Sensitivity of the high-risk asset weight with respect to the relative price. This plot shows the weight of the high-risk asset $\left(w_{A, t}\right)$ with respect to the relative price and two extreme Margrabe option parameter values. Each plot considers the weight of the high-risk asset sensitivity to a range of relative prices (from 0.6 to 1.4) and two extreme (high and low) values of high-risk asset's return volatility (top left), correlation (top right), low-risk asset's return volatility (bottom left), and time to maturity of the option (bottom right). See Table 2 for details.

\subsection{Simulation methodology}

Investors in the MBo2 solutions typically have a medium to longterm investment horizon. Typical investment horizons are one year or longer. The drawback of such a long investment horizon is that the historical data provide only a small number of evaluation samples. To overcome this problem, we follow Perold and Sharpe (1995) and Ardia et al. (2016) and backtest the MBo2 strategy by simulating $M$ artificially generated price-paths of the high-risk asset for two investment horizons: one year and five years. Based on Jegadeesh and Titman (1993), the momentum strategy requires sampling windows of at least three months to preserve the positive autocorrelation in the return series. We, therefore, apply a block-bootstrap with a block length of four and six months (for one-year and five-year investment horizons, respectively) which are sufficient to strike a balance between preserving the momentum in the returns, and allowing for heterogeneity in the simulated paths. Following Perold and Sharpe (1995) and Ardia et al. (2016), we simulate $M=10,000$ price-paths.

\subsection{Results}

Fig. 4 displays the results for the one-year investment horizon. We show the year-end value of the buy-and-hold strategy on the high-risk asset (the horizontal axis) and the weight on the high-risk asset of the MBo2 strategy (the top charts) as well as the portfolio performance (the bottom charts). Results are shown for the investment in the US market for one-year and five-year horizons.

Consider first the dependence of the high-risk asset weight on the year-end value of the high-risk asset in the two top charts. We see increasing values of the high-risk asset weight for increasing year-end values of the buy-and-hold strategy on the high-risk asset. Note that when the high-risk asset ends below one, the MBo2 strategy invests less than $50 \%$ in the high-risk asset on average (and more than 50\% in the low-risk asset). It, therefore, indicates a downside protection in the bearish market. The weights of the high-risk asset increase to about $85 \%$ when the high-risk asset ends at 1.6 in the bullish market. It implies a moderate uptrend potential of the MBo2 strategy. 

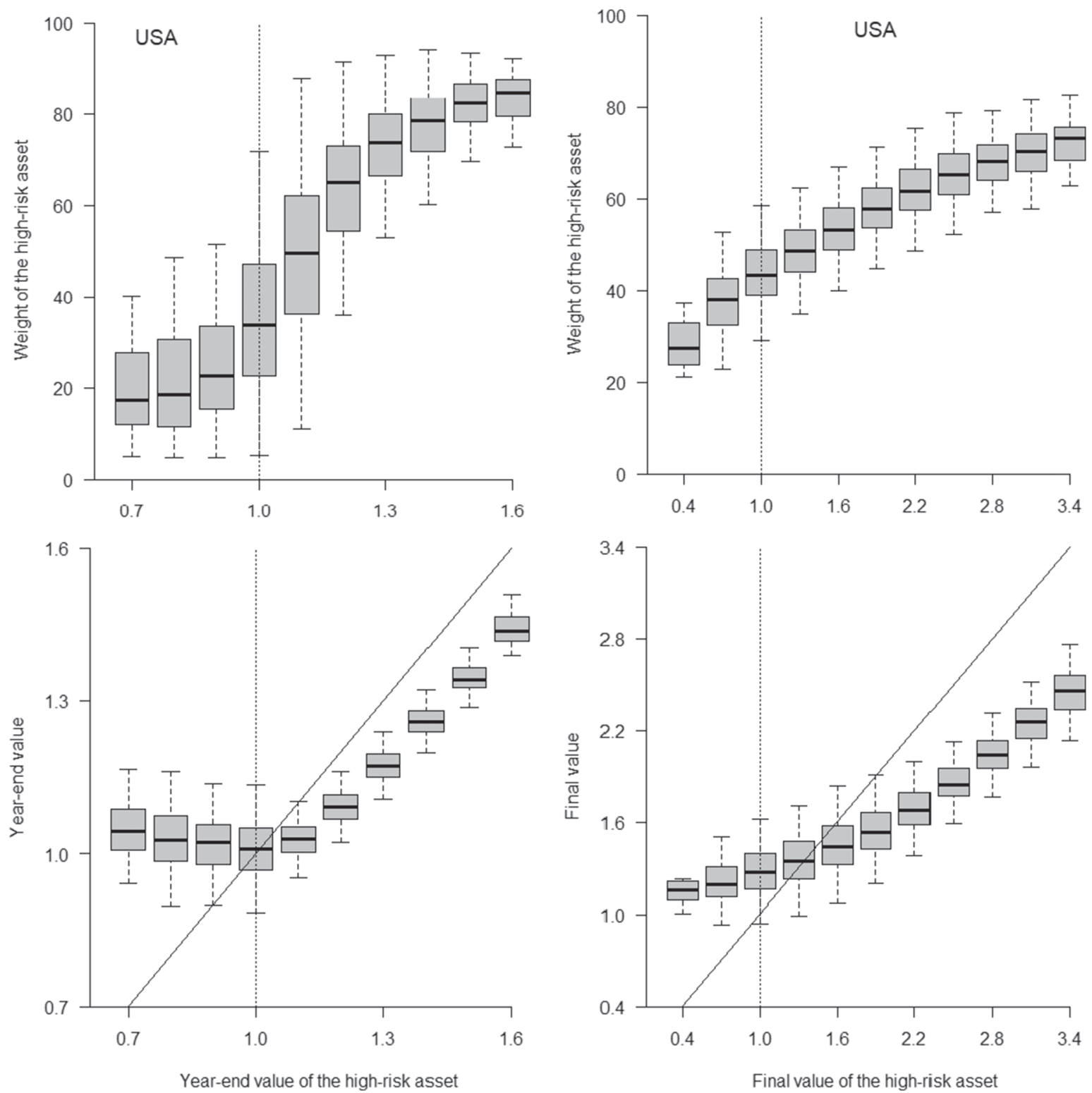

Fig. 4. Simulated portfolio composition and performance of the MBo2 strategy. These plots show the average high-risk asset weights (top plots) and the corresponding cumulative values (bottom) over one-year (left) and five-year (right) investment of the MBo2 strategy. The reference performance is the buy-and-hold strategy on the high-risk asset (full line). The strategies invest in equity and bond in the US market. Data is simulated by using the block-bootstrap technique. See Section 4 for details.

Regarding the portfolio performance (two charts at the bottom), the full line represents the year-end value of the buy-and-hold strategy on the high-risk asset. As we can see, the cumulative value is a linear function of the year-end value of the high-risk asset (the slope of the line is one). Different from the buy-and-hold strategy, the year-end value of the MBo2 strategy has a non-linear dependence on the yearend value of the high-risk asset. It implies a dependence on both of the high- and low-risk assets' price. At the extreme low year-end value of the high-risk asset (e.g., 0.7 , or $30 \%$ of drawdown, equivalently) the weight on the high-risk asset is less than $50 \%$. The portfolio value is then close to 1.05 ( $5 \%$ gain). The portfolio value gradually decreases when the year-end value of the high-risk asset increases up to one. When the year-end value of the high-risk asset is greater than one, the year-end value of the MBo2 strategy is slightly lower than those of the high-risk asset.

In Fig. 4, we also present the results of the five-year investment horizon. We see similar patterns of the weight chart and the portfolio performance compared with the performance of the one-year investment strategy. The average weights on the high-risk asset increase in stable paths in corresponding with increasing final-value of the buyand-hold strategy on the high-risk asset. Regarding the portfolio performance, when the buy-and-hold strategy on the high-risk asset yields a minimum cumulative value of 0.4 ( $60 \%$ of drawdown), the MBo2 strategy yields a cumulative value of 1.2 (20\% gain) on average. For the other cumulative values from 1.6 to 3.1 , the buy-and-hold strategy on the high-risk asset yields slightly better cumulative values than the MBo2 strategy. In the best case, the buy-and-hold strategy on the highrisk asset has a maximum cumulative value of 3.4 (240\% gain) while the cumulative value of the MBo2 strategy is 2.4 (140\% gain) on average. Similar to the one-year MBo2 investment, the five-year MBo2 strategy combines the upside potential and the downside protection. It outperforms the buy-and-hold strategy on the high-risk asset during the downtrend market and underperforms during the uptrend market. 


\section{Conclusion}

The MBo2 strategy is a tactical asset allocation strategy for setting the weights of a portfolio invested in a low-risk and high-risk asset. The rule behind the strategy is to dynamically allocate the capital on the low-risk and high-risk assets using the information on their relative price, volatilities, correlation of their returns, and the time to maturity of the exchange option. While it is somewhat popular in practice, its properties have not been studied in detail. In this paper, we provide practitioners with better insights on how the various parameters drive the weights and the performance of the MBo2 strategy.

First, we derive explicit formulae of the impact of the inputs of the strategy on the portfolio composition and the portfolio performance.
We show that the relative price and (to a lesser extent) the volatility of the high-risk asset's return are the most influential parameters driving the portfolio weight.

Second, we use the block-bootstrap technique to simulate data for studying the sensitivity of the portfolio weights and performance of the MBo2 strategy. It confirms the upside potential and the drawdown protection over the one- and five-year investment horizons.

Third, we report the results of an extensive out-of-sample evaluation for the MBo2 strategy applied to the bond-equity, real estate-equity, and world equity-emerging market equity portfolio allocation problems. The analysis shows that the MBo2 strategy yields systematically the highest return, and a lower volatility and drawdown than the buyand-hold strategy on the high-risk asset.

\section{Appendix A. Equivalent expression for the value of the MBo2 replicating portfolio}

The MBo2 strategy consists of investing in an underlying asset and buying the Margrabe option to exchange an asset for another asset. Using the valuation formula (1), we can construct a replicating portfolio by investing directly in asset $A$ and asset $B$. The value of the MBo2 replicating portfolio is equal to the sum of the price of asset $B$ and the option price to exchange asset $B$ for asset $A$ (see Rubinstein \& Leland, 1981):

$P_{\mathrm{MBo} 2, t} \equiv P_{B, t \mid t_{0}}+C_{t}^{B \rightarrow A}$.

To show that $P_{\mathrm{MBo} 2, t}=P_{A, t \mid t_{0}}+C_{t}^{A \rightarrow B}$, we first rewrite:

$$
\begin{aligned}
P_{B, t \mid t_{0}}+C_{t}^{B \rightarrow A}= & P_{B, t \mid t_{0}}+P_{A, t \mid t_{0}} \Phi\left(\frac{\ln \left(P_{A / B, t \mid t_{0}}\right)+\frac{1}{2} \sigma_{A-B, t}^{2} \tau_{t}}{\sigma_{A-B, t} \sqrt{\tau_{t}}}\right) \\
& -P_{B, t \mid t_{0}} \Phi\left(\frac{\ln \left(P_{A / B, t \mid t_{0}}\right)-\frac{1}{2} \sigma_{A-B, t}^{2} \tau_{t}}{\sigma_{A-B, t} \sqrt{\tau_{t}}}\right) .
\end{aligned}
$$

Using $\ln (x)=-\ln (1 / x)$ with $x>0$ and $\Phi(x)=1-\Phi(-x)$, we can write Eq. (A.2) as:

$$
\begin{aligned}
P_{B, t \mid t_{0}}+C_{t}^{B \rightarrow A} & =P_{A, t \mid t_{0}} \Phi\left(\frac{-\ln \left(P_{B / A, t \mid t_{0}}\right)+\frac{1}{2} \sigma_{A-B, t}^{2} \tau_{t}}{\sigma_{A-B, t} \sqrt{\tau_{t}}}\right)+P_{B, t \mid t_{0}}\left(1-\Phi\left(\frac{-\ln \left(P_{B / A, t \mid t_{0}}\right)-\frac{1}{2} \sigma_{A-B, t}^{2} \tau_{t}}{\sigma_{A-B, t} \sqrt{\tau_{t}}}\right)\right) \\
& =P_{A, t \mid t_{0}}\left(1-\Phi\left(\frac{\ln \left(P_{B / A, t \mid t_{0}}\right)-\frac{1}{2} \sigma_{A-B, t}^{2} \tau_{t}}{\sigma_{A-B, t} \sqrt{\tau_{t}}}\right)\right)+P_{B, t \mid t_{0}} \Phi\left(\frac{\ln \left(P_{B / A, t \mid t_{0}}\right)+\frac{1}{2} \sigma_{A-B, t}^{2} \tau_{t}}{\sigma_{A-B, t} \sqrt{\tau_{t}}}\right) \\
& =P_{A, t \mid t_{0}}+P_{B, t \mid t_{0}} \Phi\left(\frac{\ln \left(P_{B / A, t \mid t_{0}}\right)+\frac{1}{2} \sigma_{A-B, t}^{2} \tau_{t}}{\sigma_{A-B, t} \sqrt{\tau_{t}}}\right)-P_{A, t \mid t_{0}} \Phi\left(\frac{\ln \left(P_{B / A, t \mid t_{0}}\right)-\frac{1}{2} \sigma_{A-B, t}^{2} \tau_{t}}{\sigma_{A-B, t} \sqrt{\tau_{t}}}\right) \\
& =P_{A, t \mid t_{0}}+C_{t}^{A \rightarrow B} .
\end{aligned}
$$

The value of the MBo2 replicating portfolio is:

$P_{\mathrm{MBo}, t}=P_{A, t \mid t_{0}} \Phi\left(d_{1, t}\right)+P_{B, t \mid t_{0}}\left(1-\Phi\left(d_{2, t}\right)\right)$.

\section{Appendix B. Composition of the MBo2 replicating portfolio}

Given the value of the Margrabe replicating portfolio in Appendix A, the question is how many units of asset $A\left(n_{A, t}\right)$ and asset $B\left(n_{B, t}\right)$ do we need to buy to have a portfolio with the value equal to the value of the MBo2 replicating portfolio? From equal-value conditions, we have:

$P_{A, t} n_{A, t}+P_{B, t} n_{B, t}=P_{\mathrm{MBo} 2, t}=P_{A, t \mid t_{0}} \Phi\left(d_{1, t}\right)+P_{B, t \mid t_{0}}\left(1-\Phi\left(d_{2, t}\right)\right)$.

We have that $P_{A, t}=P_{A, t \mid t_{0}} P_{A, t_{0}}$ and $P_{B, t}=P_{B, t \mid t_{0}} P_{B, t_{0}}$, which implies:

$P_{A, t_{0}} n_{A, t}=\Phi\left(d_{1, t}\right)$

$P_{B, t_{0}} n_{B, t}=1-\Phi\left(d_{2, t}\right)$.

The weight of asset $A$ in the MBo2 replicating portfolio is:

$w_{A, t} \equiv \frac{P_{A, t} n_{A, t}}{P_{A, t} n_{A, t}+P_{B, t} n_{B, t}}=\frac{P_{A, t \mid t_{0}} \Phi\left(d_{1, t}\right)}{P_{A, t \mid t_{0}} \Phi\left(d_{1, t}\right)+P_{B, t \mid t_{0}}\left(1-\Phi\left(d_{2, t}\right)\right)}=\frac{P_{A / B, t \mid t_{0}} \Phi\left(d_{1, t}\right)}{P_{A / B, t \mid t_{0}} \Phi\left(d_{1, t}\right)+\left(1-\Phi\left(d_{2, t}\right)\right)}$, 
and the weight of asset $B$ in the MBo2 replicating portfolio is:

$w_{B, t} \equiv \frac{P_{B, t} n_{B, t}}{P_{A, t} n_{A, t}+P_{B, t} n_{B, t}}=\frac{P_{B, t \mid t_{0}}\left(1-\Phi\left(d_{2, t}\right)\right)}{P_{A, t \mid t_{0}} \Phi\left(d_{1, t}\right)+P_{B, t \mid t_{0}}\left(1-\Phi\left(d_{2, t}\right)\right)}=\frac{\left(1-\Phi\left(d_{2, t}\right)\right)}{P_{A / B, t \mid t_{0}} \Phi\left(d_{1, t}\right)+\left(1-\Phi\left(d_{2, t}\right)\right)}$.

Note that $w_{B, t}=1-w_{A, t}$.

To prove Eq. (3), we further recall that under the Black-Scholes assumptions, we have that, at time $t$, the standardized variable:

$z \equiv \frac{\ln \left(\frac{P_{A / B, T \mid t}}{P_{A / B, t \mid t_{0}}}\right)+\frac{\sigma_{A-B, t}^{2}}{2} \tau_{t}}{\sigma_{A-B, t} \sqrt{\tau_{t}}} \sim N(0,1)$

At maturity, the expected value of the relative price conditional on the relative price is greater than one is:

$$
\begin{aligned}
\mathbb{E}\left[P_{A / B, T \mid t} \mid P_{A / B, T \mid t}>1\right] & =\mathbb{E}\left[P_{A / B, T \mid t} \mid \ln \left(P_{A / B, T \mid t}\right)>0\right] \\
& =\int_{\ln \left(P_{A / B, T \mid t}\right)=0}^{+\infty} P_{A / B, T \mid t} f\left(\ln \left(P_{A / B, T \mid t}\right)\right) d\left(\ln \left(P_{A / B, T \mid t}\right)\right) \\
& =P_{A / B, t \mid t_{0}} \frac{1}{\sqrt{2 \pi}} \int_{-d_{2, t}}^{+\infty} e^{-\frac{\left(z-\sigma_{A-B, t} \sqrt{\tau_{t}}\right)^{2}}{2}} d z \\
& =P_{A / B, t \mid t_{0}} \frac{1}{\sqrt{2 \pi}} \int_{y=-d_{2, t}-\sigma_{A-B, t} \sqrt{\tau_{t}}}^{+\infty} e^{-\frac{y^{2}}{2}} d y \\
& =P_{A / B, t \mid t_{0}} \frac{1}{\sqrt{2 \pi}} \int_{-d_{1, t}}^{+\infty} e^{-\frac{y^{2}}{2}} d y \\
& =P_{A / B, t \mid t_{0}}\left(1-\Phi\left(-d_{1, t}\right)\right)=P_{A / B, t \mid t_{0}} \Phi\left(d_{1, t}\right)
\end{aligned}
$$

where $f(x)$ is the probability density function of the normal random variable $\ln \left(P_{A / B, T \mid t}\right)$ with mean $\ln \left(P_{A / B, t \mid t_{0}}\right)-\frac{\sigma_{A-B, t}^{2}}{2} \tau_{t}$ and variance $\sigma_{A-B, t}^{2} \tau_{t}$. Note that we use $y=z-\sigma_{A-B, t} \sqrt{\tau_{t}}$ and $d y=d z$ in the fifth equation.

The probability that the relative price will be higher than one at maturity is:

$$
\begin{aligned}
\mathbb{P}\left[P_{A / B, T \mid t}>1\right] & =\mathbb{P}\left[\ln \left(P_{A / B, T \mid t}\right)>0\right]=\int_{\ln \left(P_{A / B, T \mid t}\right)=0}^{+\infty} f\left(\ln \left(P_{A / B, T \mid t}\right)\right) d\left(\ln \left(P_{A / B, T \mid t}\right)\right) \\
& =\int_{z=-d_{2, t}}^{+\infty} f(z) d z=1-\Phi\left(-d_{2, t}\right)=\Phi\left(d_{2, t}\right) .
\end{aligned}
$$

Then the weight of asset $A$ in Eq. (B.4) can be rewritten as:

$$
w_{A, t}=\frac{\mathbb{E}\left[P_{A / B, T \mid t} \mid P_{A / B, T \mid t}>1\right]}{\mathbb{E}\left[P_{A / B, T \mid t} \mid P_{A / B, T \mid t}>1\right]+\mathbb{P}\left[P_{A / B, T \mid t}<1\right]} .
$$

The weight of asset $B$ is:

$w_{B, t}=\frac{\mathbb{P}\left(P_{A / B, T \mid t}<1\right)}{\mathbb{E}\left[P_{A / B, T \mid t} \mid P_{A / B, T \mid t}>1\right]+\mathbb{P}\left[P_{A / B, T \mid t}<1\right]}$.

\section{Appendix C. Sensitivity of $\boldsymbol{w}_{A, t}$ in the special case where $P_{A / B, t \mid t_{0}}=1$}

In the special case where $P_{A / B, t \mid t_{0}}=1$, we have $d_{1, t}=-d_{2, t}$ and $\Phi\left(d_{1, t}\right)=1-\Phi\left(d_{2, t}\right)$. From Eq. (3), the weight of the high-risk asset is then:

$w_{A, t}=\frac{P_{A / B, t \mid t_{0}} \Phi\left(d_{1, t}\right)}{P_{A / B, t \mid t_{0}} \Phi\left(d_{1, t}\right)+\left(1-\Phi\left(d_{2, t}\right)\right)}=\frac{\Phi\left(d_{1, t}\right)}{\Phi\left(d_{1, t}\right)+\Phi\left(d_{1, t}\right)}=\frac{1}{2}$.

\section{Appendix D. Sensitivity of $\boldsymbol{w}_{A, t}$ with respect to $P_{A / B, t \mid t_{0}}$}

The partial derivative of $\Phi\left(d_{i, t}\right)(i=1,2)$ with respect to $P_{A / B, t \mid t_{0}}$ is:

$\frac{\partial \Phi\left(d_{i, t}\right)}{\partial P_{A / B, t \mid t_{0}}}=\phi\left(d_{i, t}\right) \frac{1}{P_{A / B, t \mid t_{0}} \sigma_{A-B, t} \sqrt{\tau_{t}}} \geq 0$

Then the partial derivative of $w_{A, t}$ with respect to $P_{A / B, t \mid t_{0}}$ is: 


$$
\begin{aligned}
& \frac{\partial w_{A, t}}{\partial P_{A / B, t \mid t_{0}}}=\frac{1}{\left[P_{A / B, t \mid t_{0}} \Phi\left(d_{1, t}\right)+\left(1-\Phi\left(d_{2, t}\right)\right)\right]^{2}} \times\left[( P _ { A / B , t | t _ { 0 } } \Phi ( d _ { 1 , t } ) + ( 1 - \Phi ( d _ { 2 , t } ) ) ) \left(\Phi\left(d_{1, t}\right)\right.\right. \\
& \left.\left.+P_{A / B, t \mid t_{0}} \frac{\partial \Phi\left(d_{1, t}\right)}{\partial P_{A / B, t \mid t_{0}}}\right)-P_{A / B, t \mid t_{0}} \Phi\left(d_{1, t}\right)\left(\Phi\left(d_{1, t}\right)+P_{A / B, t \mid t_{0}} \frac{\partial \Phi\left(d_{1, t}\right)}{\partial P_{A / B, t \mid t_{0}}}-\frac{\partial \Phi\left(d_{2, t}\right)}{\partial P_{A / B, t \mid t_{0}}}\right)\right] \\
& =\frac{1}{\left[P_{A / B, t \mid t_{0}} \Phi\left(d_{1, t}\right)+\left(1-\Phi\left(d_{2, t}\right)\right)\right]^{2}} \times\left[P_{A / B, t \mid t_{0}} \Phi^{2}\left(d_{1, t}\right)+P_{A / B, t}^{2} \Phi\left(d_{1, t}\right) \frac{\partial \Phi\left(d_{1, t}\right)}{\partial P_{A / B, t \mid t_{0}}}\right. \\
& +\left(1-\Phi\left(d_{2, t}\right)\right) \Phi\left(d_{1, t}\right)+P_{A / B, t \mid t_{0}}\left(1-\Phi\left(d_{2, t}\right)\right) \frac{\partial \Phi\left(d_{1, t}\right)}{\partial P_{A / B, t \mid t_{0}}}-P_{A / B, t \mid t_{0}} \Phi^{2}\left(d_{1, t}\right) \\
& \left.-P_{A / B, t}^{2} \Phi\left(d_{1, t}\right) \frac{\partial \Phi\left(d_{1, t}\right)}{\partial P_{A / B, t \mid t_{0}}}+P_{A / B, t \mid t_{0}} \Phi\left(d_{1, t}\right) \frac{\partial \Phi\left(d_{2, t}\right)}{\partial P_{A / B, t \mid t_{0}}}\right] \\
& =\frac{\left(1-\Phi\left(d_{2, t}\right)\right) \Phi\left(d_{1, t}\right)+P_{A / B, t \mid t_{0}}\left(1-\Phi\left(d_{2, t}\right)\right) \frac{\partial \Phi\left(d_{1, t}\right)}{\partial P_{A / B, t \mid t_{0}}}+P_{A / B, t \mid t_{0}} \frac{\partial \Phi\left(d_{2, t}\right)}{\partial P_{A / B, t \mid t_{0}}}}{\left[P_{A / B, t \mid t_{0}} \Phi\left(d_{1, t}\right)+\left(1-\Phi\left(d_{2, t}\right)\right)\right]^{2}} \\
& =\frac{\left(1-\Phi\left(d_{2, t}\right)\right) \Phi\left(d_{1, t}\right)+\left(1-\Phi\left(d_{2, t}\right)\right) \phi\left(d_{1, t}\right) \frac{1}{\sigma_{A-B, t} \sqrt{\tau_{t}}}+\phi\left(d_{2, t}\right) \frac{1}{\sigma_{A-B, t \sqrt{t}}}}{\left[P_{A / B, t \mid t_{0}} \Phi\left(d_{1, t}\right)+\left(1-\Phi\left(d_{2, t}\right)\right)\right]^{2}} \\
& =\frac{\left(1-\Phi\left(d_{2, t}\right)\right) \Phi\left(d_{1, t}\right)+\frac{1}{\sigma_{A-B, t \sqrt{t}}}\left[\left(1-\Phi\left(d_{2, t}\right)\right) \phi\left(d_{1, t}\right)+\phi\left(d_{2, t}\right)\right]}{\left[P_{A / B, t \mid t_{0}} \Phi\left(d_{1, t}\right)+\left(1-\Phi\left(d_{2, t}\right)\right)\right]^{2}} \text {. }
\end{aligned}
$$

Expression (D.2) shows the non-negative relation between the relative price and the weight of the high-risk asset in the MBo2 replicating portfolio. The higher the relative price, the higher its weight in the MBo2 replicating portfolio.

\section{Appendix E. Sensitivity of $w_{A, t}$ with respect to $\sigma_{A, t}$}

The partial derivative of $\Phi\left(d_{1, t}\right)$ with respect to $\sigma_{A, t}$ is:

$$
\begin{aligned}
\frac{\partial \Phi\left(d_{1, t}\right)}{\partial \sigma_{A, t}} & =\phi\left(d_{1, t}\right)\left[-\frac{\ln \left(P_{A / B, t \mid t_{0}}\right)}{\sigma_{A-B, t}^{2} \tau_{t}} \frac{1}{2} \sqrt{\tau_{t}}\left(2 \sigma_{A, t}-2 \sigma_{B, t} \rho_{A, B, t}\right)+\frac{1}{2} \sqrt{\tau_{t}}\left(2 \sigma_{A, t}-2 \sigma_{B, t} \rho_{A, B, t}\right)\right] \\
& =\phi\left(d_{1, t}\right) \sqrt{\tau_{t}}\left(\sigma_{A, t}-\sigma_{B, t} \rho_{A, B, t}\right)\left(1-\frac{\ln \left(P_{A / B, t \mid t_{0}}\right)}{\sigma_{A-B, t}^{2} \tau_{t}}\right) .
\end{aligned}
$$

The partial derivative of $\Phi\left(d_{2, t}\right)$ with respect to $\sigma_{A, t}$ is:

$$
\begin{aligned}
\frac{\partial \Phi\left(d_{2, t}\right)}{\partial \sigma_{A, t}} & =\phi\left(d_{2, t}\right)\left[-\frac{\ln \left(P_{A / B, t \mid t_{0}}\right)}{\sigma_{A-B, t}^{2} \tau_{t}} \frac{1}{2} \sqrt{\tau_{t}}\left(2 \sigma_{A, t}-2 \sigma_{B, t} \rho_{A, B, t}\right)-\frac{1}{2} \sqrt{\tau_{t}}\left(2 \sigma_{A, t}-2 \sigma_{B, t} \rho_{A, B, t}\right)\right] \\
& =-\phi\left(d_{2, t}\right) \sqrt{\tau_{t}}\left(\sigma_{A, t}-\sigma_{B, t} \rho_{A, B, t}\right)\left(1+\frac{\ln \left(P_{A / B, t \mid t_{0}}\right)}{\sigma_{A-B, t}^{2} \tau_{t}}\right) .
\end{aligned}
$$

Then, the partial derivative of $w_{A, t}$ with respect to $\sigma_{A, t}$ is:

$$
\begin{aligned}
\frac{\partial w_{A, t}}{\partial \sigma_{A, t}=} & \frac{1}{\left[P_{A / B, t \mid t_{0}} \Phi\left(d_{1, t}\right)+\left(1-\Phi\left(d_{2, t}\right)\right)\right]^{2}}\left[\left(P_{A / B, t \mid t_{0}} \Phi\left(d_{1, t}\right)+\left(1-\Phi\left(d_{2, t}\right)\right)\right) P_{A / B, t \mid t_{0}} \frac{\partial \Phi\left(d_{1, t}\right)}{\partial \sigma_{A, t}}\right. \\
& \left.-P_{A / B, t \mid t_{0}} \Phi\left(d_{1, t}\right)\left(\frac{P_{A / B, t \mid t_{0}} \partial \Phi\left(d_{1, t}\right)}{\partial \sigma_{A, t}}-\frac{\partial \Phi\left(d_{2, t}\right)}{\partial \sigma_{A, t}}\right)\right] \\
= & \frac{1}{\left[P_{A / B, t \mid t_{0}} \Phi\left(d_{1, t}\right)+\left(1-\Phi\left(d_{2, t}\right)\right)\right]^{2}}\left[P_{A / B, t \mid t_{0}}^{2} \Phi\left(d_{1, t}\right) \frac{\partial \Phi\left(d_{1, t}\right)}{\partial \sigma_{A, t}}+\left(1-\Phi\left(d_{2, t}\right)\right) P_{A / B, t \mid t_{0}} \frac{\partial \Phi\left(d_{1, t}\right)}{\partial \sigma_{A, t}}\right. \\
& \left.-P_{A / B, t \mid t_{0}}^{2} \Phi\left(d_{1, t}\right) \frac{\partial \Phi\left(d_{1, t}\right)}{\partial \sigma_{A, t}}+P_{A / B, t \mid t_{0}} \Phi\left(d_{1, t}\right) \frac{\partial \Phi\left(d_{2, t}\right)}{\partial \sigma_{A, t}}\right] \\
= & \frac{1}{\left[P_{A / B, t \mid t_{0}} \Phi\left(d_{1, t}\right)+\left(1-\Phi\left(d_{2, t}\right)\right)\right]^{2}}\left[\left(1-\Phi\left(d_{2, t}\right)\right) P_{A / B, t \mid t_{0}} \frac{\partial \Phi\left(d_{1, t}\right)}{\partial \sigma_{A, t}}+P_{A / B, t \mid t_{0}} \Phi\left(d_{1, t}\right) \frac{\partial \Phi\left(d_{2, t}\right)}{\partial \sigma_{A, t}}\right] \\
= & \frac{P_{A / B, t \mid t_{0}} \sqrt{\tau_{t}}\left(\sigma_{A, t}-\sigma_{B, t} \rho_{A, B, t}\right)}{\left[P_{A / B, t \mid t_{0}} \Phi\left(d_{1, t}\right)+\left(1-\Phi\left(d_{2, t}\right)\right)\right]^{2}} \\
& \times\left[\left(1-\Phi\left(d_{2, t}\right)\right) \phi\left(d_{1, t}\right)\left(1-\frac{\ln \left(P_{A / B, t \mid t_{0}}\right)}{\sigma_{A-B, t}^{2} \tau_{t}}\right)-\Phi\left(d_{1, t}\right) \phi\left(d_{2, t}\right)\left(1+\frac{\ln \left(P_{A / B, t \mid t_{0}}\right)}{\sigma_{A-B, t}^{2} \tau_{t}}\right)\right] .
\end{aligned}
$$


Appendix F. Sensitivity of $w_{A, t}$ with respect to $\sigma_{B, t}$

The partial derivative of $\Phi\left(d_{1, t}\right)$ with respect to $\sigma_{B, t}$ is:

$$
\begin{aligned}
\frac{\partial \Phi\left(d_{1, t}\right)}{\partial \sigma_{B, t}} & =\phi\left(d_{1, t}\right)\left[-\frac{\ln \left(P_{A / B, t \mid t_{0}}\right)}{\sigma_{A-B, t}^{2} \tau_{t}} \frac{1}{2} \sqrt{\tau_{t}}\left(2 \sigma_{B, t}-2 \sigma_{A, t} \rho_{A, B, t}\right)+\frac{1}{2} \sqrt{\tau_{t}}\left(2 \sigma_{B, t}-2 \sigma_{A, t} \rho_{A, B, t}\right)\right] \\
& =\phi\left(d_{1, t}\right) \sqrt{\tau_{t}}\left(\sigma_{B, t}-\sigma_{A, t} \rho_{A, B, t}\right)\left(1-\frac{\ln \left(P_{A / B, t \mid t_{0}}\right)}{\sigma_{A-B, t}^{2} \tau_{t}}\right) .
\end{aligned}
$$

The partial derivative of $\Phi\left(d_{2, t}\right)$ with respect to $\sigma_{B, t}$ is:

$$
\begin{aligned}
\frac{\partial \Phi\left(d_{2, t}\right)}{\partial \sigma_{B, t}} & =\phi\left(d_{2, t}\right)\left[-\frac{\ln \left(P_{A / B, t \mid t_{0}}\right)}{\sigma_{A-B, t}^{2} \tau_{t}} \frac{1}{2} \sqrt{\tau_{t}}\left(2 \sigma_{B, t}-2 \sigma_{A, t} \rho_{A, B, t}\right)-\frac{1}{2} \sqrt{\tau_{t}}\left(2 \sigma_{B, t}-2 \sigma_{A, t} \rho_{A, B, t}\right)\right] \\
& =-\phi\left(d_{2, t}\right) \sqrt{\tau_{t}}\left(\sigma_{B, t}-\sigma_{A, t} \rho_{A, B, t}\right)\left(1+\frac{\ln \left(P_{A / B, t \mid t_{0}}\right)}{\sigma_{A-B, t}^{2} \tau_{t}}\right) .
\end{aligned}
$$

Then, the partial derivative of $w_{A, t}$ with respect to $\sigma_{B, t}$ is:

$$
\begin{aligned}
\frac{\partial w_{A, t}}{\partial \sigma_{B, t}}= & \frac{1}{\left[P_{A / B, t \mid t_{0}} \Phi\left(d_{1, t}\right)+\left(1-\Phi\left(d_{2, t}\right)\right)\right]^{2}}\left[\left(P_{A / B, t \mid t_{0}} \Phi\left(d_{1, t}\right)+\left(1-\Phi\left(d_{2, t}\right)\right)\right) P_{A / B, t \mid t_{0}} \frac{\partial \Phi\left(d_{1, t}\right)}{\partial \sigma_{A, t}}\right. \\
& \left.-P_{A / B, t \mid t_{0}} \Phi\left(d_{1, t}\right)\left(\frac{P_{A / B, t \mid t_{0}} \partial \Phi\left(d_{1, t}\right)}{\partial \sigma_{A, t}}-\frac{\partial \Phi\left(d_{2, t}\right)}{\partial \sigma_{A, t}}\right)\right] \\
= & \frac{1}{\left[P_{A / B, t \mid t_{0}} \Phi\left(d_{1, t}\right)+\left(1-\Phi\left(d_{2, t}\right)\right)\right]^{2}}\left[P_{A / B, t \mid t_{0}}^{2} \Phi\left(d_{1, t}\right) \frac{\partial \Phi\left(d_{1, t}\right)}{\partial \sigma_{B, t}}+\left(1-\Phi\left(d_{2, t}\right)\right) P_{A / B, t \mid t_{0}} \frac{\partial \Phi\left(d_{1, t}\right)}{\partial \sigma_{B, t}}\right. \\
& \left.-P_{A / B, t \mid t_{0}}^{2} \Phi\left(d_{1, t}\right) \frac{\partial \Phi\left(d_{1, t}\right)}{\partial \sigma_{B, t}}+P_{A / B, t \mid t_{0}} \Phi\left(d_{1, t}\right) \frac{\partial \Phi\left(d_{2, t}\right)}{\partial \sigma_{B, t}}\right] \\
= & \frac{1}{\left[P_{A / B, t \mid t_{0}} \Phi\left(d_{1, t}\right)+\left(1-\Phi\left(d_{2, t}\right)\right)\right]^{2}}\left[\left(1-\Phi\left(d_{2, t}\right)\right) P_{A / B, t \mid t_{0}} \frac{\partial \Phi\left(d_{1, t}\right)}{\partial \sigma_{B, t}}+P_{A / B, t \mid t_{0}} \Phi\left(d_{1, t}\right) \frac{\partial \Phi\left(d_{2, t}\right)}{\partial \sigma_{B, t}}\right] \\
= & \frac{P_{A / B, t \mid t_{0}} \sqrt{\tau_{t}}\left(\sigma_{B, t}-\sigma_{A, t} \rho_{A, B, t}\right)}{\left[P_{A / B, t \mid t_{0}} \Phi\left(d_{1, t}\right)+\left(1-\Phi\left(d_{2, t}\right)\right)\right]^{2}} \\
& \times\left[\left(1-\Phi\left(d_{2, t}\right)\right) \phi\left(d_{1, t}\right)\left(1-\frac{\ln \left(P_{A / B, t \mid t_{0}}\right)}{\sigma_{A-B, t}^{2} \tau_{t}}\right)-\Phi\left(d_{1, t}\right) \phi\left(d_{2, t}\right)\left(1+\frac{\ln \left(P_{A / B, t \mid t_{0}}\right)}{\sigma_{A-B, t}^{2} \tau_{t}}\right)\right] .
\end{aligned}
$$

\section{Appendix G. Sensitivity of $w_{A, t}$ with respect to $\rho_{A, B, t}$}

The partial derivative of $\Phi\left(d_{1, t}\right)$ with respect to $\rho_{A, B, t}$ is:

$$
\begin{aligned}
\frac{\partial \Phi\left(d_{1, t}\right)}{\partial \rho_{A, B, t}} & =\phi\left(d_{1, t}\right)\left[-\frac{\ln \left(P_{A / B, t \mid t_{0}}\right)}{\sigma_{A-B, t}^{2} \tau_{t}} \frac{1}{2} \sqrt{\tau_{t}}\left(-2 \sigma_{A, t} \sigma_{B, t}\right)+\frac{1}{2} \sqrt{\tau_{t}}\left(-2 \sigma_{A, t} \sigma_{B, t}\right)\right] \\
& =\phi\left(d_{1, t}\right) \sqrt{\tau_{t}} \sigma_{A, t} \sigma_{B, t}\left(\frac{\ln \left(P_{A / B, t \mid t_{0}}\right)}{\sigma_{A-B, t}^{2} \tau_{t}}-1\right) .
\end{aligned}
$$

The partial derivative of $\Phi\left(d_{2, t}\right)$ with respect to $\rho_{A, B, t}$ is:

$$
\begin{aligned}
\frac{\partial \Phi\left(d_{2, t}\right)}{\partial \rho_{A, B, t}} & =\phi\left(d_{2, t}\right)\left[-\frac{\ln \left(P_{A / B, t \mid t_{0}}\right)}{\sigma_{A-B, t}^{2} \tau_{t}} \frac{1}{2} \sqrt{\tau_{t}}\left(-2 \sigma_{A, t} \sigma_{B, t}\right)-\frac{1}{2} \sqrt{\tau_{t}}\left(-2 \sigma_{A, t} \sigma_{B, t}\right)\right] \\
& =\phi\left(d_{2, t}\right) \sqrt{\tau_{t}} \sigma_{A, t} \sigma_{B, t}\left(\frac{\ln \left(P_{A / B, t \mid t_{0}}\right)}{\sigma_{A-B, t}^{2} \tau_{t}}+1\right) .
\end{aligned}
$$

Then we can rewrite the sensitivity of $w_{A, t}$ with respect to $\rho_{A, B, t}$ as: 


$$
\begin{aligned}
\frac{\partial w_{A, t}}{\partial \rho_{A, B, t}=} & \frac{1}{\left[P_{A / B, t \mid t_{0}} \Phi\left(d_{1, t}\right)+\left(1-\Phi\left(d_{2, t}\right)\right)\right]^{2}}\left[\left(P_{A / B, t \mid t_{0}} \Phi\left(d_{1, t}\right)+1-\Phi\left(d_{2, t}\right)\right) P_{A / B, t \mid t_{0}} \frac{\partial \Phi\left(d_{1, t}\right)}{\partial \rho_{A, B, t}}\right. \\
& \left.-P_{A / B, t \mid t_{0}} \Phi\left(d_{1, t}\right)\left(\frac{P_{A / B, t \mid t_{0}} \partial \Phi\left(d_{1, t}\right)}{\partial \rho_{A, B, t}}-\frac{\partial \Phi\left(d_{2, t}\right)}{\partial \rho_{A, B, t}}\right)\right] \\
= & \frac{1}{\left[P_{A / B, t \mid t_{0}} \Phi\left(d_{1, t}\right)+\left(1-\Phi\left(d_{2, t}\right)\right)\right]^{2}}\left[P_{A / B, t \mid t_{0}}^{2} \Phi\left(d_{1, t}\right) \frac{\partial \Phi\left(d_{1, t}\right)}{\partial \rho_{A, B, t}}+\left(1-\Phi\left(d_{2, t}\right)\right) P_{A / B, t \mid t_{0}} \frac{\partial \Phi\left(d_{1, t}\right)}{\partial \rho_{A, B, t}}\right. \\
& \left.-P_{A / B, t \mid t_{0}}^{2} \Phi\left(d_{1, t}\right) \frac{\partial \Phi\left(d_{1, t}\right)}{\partial \rho_{A, B, t}}+P_{A / B, t \mid t_{0}} \Phi\left(d_{1, t}\right) \frac{\partial \Phi\left(d_{2, t}\right)}{\partial \rho_{A, B, t}}\right] \\
= & \frac{1}{\left[P_{A / B, t \mid t_{0}} \Phi\left(d_{1, t}\right)+\left(1-\Phi\left(d_{2, t}\right)\right)\right]^{2}}\left[\left(1-\Phi\left(d_{2, t}\right)\right) P_{A / B, t \mid t_{0}} \frac{\partial \Phi\left(d_{1, t}\right)}{\partial \rho_{A, B, t}}+P_{A / B, t \mid t_{0}} \Phi\left(d_{1, t}\right) \frac{\partial \Phi\left(d_{2, t}\right)}{\partial \rho_{A, B, t}}\right] \\
= & \frac{P_{A / B, t \mid t_{0}} \sqrt{\tau_{t} \sigma_{A, t} \sigma_{B, t}}}{\left[P_{A / B, t \mid t_{0}} \Phi\left(d_{1, t}\right)+\left(1-\Phi\left(d_{2, t}\right)\right)\right]^{2}} \\
& \times\left[\left(1-\Phi\left(d_{2, t}\right)\right) \phi\left(d_{1, t}\right)\left(\frac{\ln \left(P_{A / B, t \mid t_{0}}\right)}{\sigma_{A-B, t}^{2} \tau_{t}}-1\right)+\Phi\left(d_{1, t}\right) \phi\left(d_{2, t}\right)\left(1+\frac{\ln \left(P_{A / B, t \mid t_{0}}\right)}{\sigma_{A-B, t}^{2} \tau_{t}}\right)\right] .
\end{aligned}
$$

\section{Appendix H. Sensitivity of $w_{A, t}$ with respect to $\tau_{t}$}

The partial derivative of $\Phi\left(d_{1, t}\right)$ with respect to $\tau_{t}$ is:

$$
\begin{aligned}
\frac{\partial \Phi\left(d_{1, t}\right)}{\partial \tau_{t}} & =\phi\left(d_{1, t}\right)\left[-\frac{\ln \left(P_{A / B, t \mid t_{0}}\right)}{2 \sigma_{A-B, t} \sqrt{\left(\tau_{t}\right)^{3}}}+\frac{1}{2} \sigma_{A-B, t} \sqrt{\tau_{t}}\right] \\
& =\phi\left(d_{1, t}\right) \frac{1}{2} \sqrt{\tau_{t}} \sigma_{A-B, t}\left(1-\frac{\ln \left(P_{A / B, t \mid t_{0}}\right)}{\sigma_{A-B, t}^{2} \tau_{t}}\right) .
\end{aligned}
$$

The partial derivative of $\Phi\left(d_{2, t}\right)$ with respect to $\tau_{t}$ is:

$$
\begin{aligned}
\frac{\partial \Phi\left(d_{2, t}\right)}{\partial \tau_{t}} & =\phi\left(d_{2, t}\right)\left[-\frac{\ln \left(P_{A / B, t \mid t_{0}}\right)}{2 \sigma_{A-B, t} \sqrt{\left(\tau_{t}\right)^{3}}}-\frac{1}{2} \sigma_{A-B, t} \sqrt{\tau_{t}}\right] \\
& =-\phi\left(d_{2, t}\right) \frac{1}{2} \sqrt{\tau_{t}} \sigma_{A-B, t}\left(1+\frac{\ln \left(P_{A / B, t \mid t_{0}}\right)}{\sigma_{A-B, t}^{2} \tau_{t}}\right) .
\end{aligned}
$$

Then, the partial derivative of $w_{A, t}$ with respect to the time to maturity of the Margrabe option is:

$$
\begin{aligned}
\frac{\partial w_{A, t}}{\partial \tau_{t}}= & \frac{1}{\left[P_{A / B, t \mid t_{0}} \Phi\left(d_{1, t}\right)+\left(1-\Phi\left(d_{2, t}\right)\right)\right]^{2}}\left[\left(P_{A / B, t \mid t_{0}} \Phi\left(d_{1, t}\right)+\left(1-\Phi\left(d_{2, t}\right)\right)\right) P_{A / B, t \mid t_{0}} \frac{\partial \Phi\left(d_{1, t}\right)}{\partial \tau_{t}}\right. \\
& \left.-P_{A / B, t \mid t_{0}} \Phi\left(d_{1, t}\right)\left(\frac{P_{A / B, t \mid t_{0}} \partial \Phi\left(d_{1, t}\right)}{\partial \tau_{t}}-\frac{\partial \Phi\left(d_{2, t}\right)}{\partial \tau_{t}}\right)\right] \\
= & \frac{1}{\left[P_{A / B, t \mid t_{0}} \Phi\left(d_{1, t}\right)+\left(1-\Phi\left(d_{2, t}\right)\right)\right]^{2}}\left[P_{A / B, t \mid t_{0}}^{2} \Phi\left(d_{1, t}\right) \frac{\partial \Phi\left(d_{1, t}\right)}{\partial \tau_{t}}+\left(1-\Phi\left(d_{2, t}\right)\right) P_{A / B, t \mid t_{0}} \frac{\partial \Phi\left(d_{1, t}\right)}{\partial \tau_{t}}\right. \\
& \left.-P_{A / B, t \mid t_{0}}^{2} \Phi\left(d_{1, t}\right) \frac{\partial \Phi\left(d_{1, t}\right)}{\partial \tau_{t}}+P_{A / B, t \mid t_{0}} \Phi\left(d_{1, t}\right) \frac{\partial \Phi\left(d_{2, t}\right)}{\partial \tau_{t}}\right] \\
= & \frac{1}{\left[P_{A / B, t \mid t_{0}} \Phi\left(d_{1, t}\right)+\left(1-\Phi\left(d_{2, t}\right)\right)\right]^{2}}\left[\left(1-\Phi\left(d_{2, t}\right)\right) P_{A / B, t \mid t_{0}} \frac{\partial \Phi\left(d_{1, t}\right)}{\partial \tau_{t}}+P_{A / B, t \mid t_{0}} \Phi\left(d_{1, t}\right) \frac{\partial \Phi\left(d_{2, t}\right)}{\partial \tau_{t}}\right] \\
& \frac{\frac{1}{2} P_{A / B, t \mid t_{0}} \sqrt{\tau_{t}} \sigma_{A-B, t}}{\left[P_{A / B, t \mid t_{0}} \Phi\left(d_{1, t}\right)+\left(1-\Phi\left(d_{2, t}\right)\right)\right]^{2}} \\
& \times\left[\left(1-\Phi\left(d_{2, t}\right)\right) \phi\left(d_{1, t}\right)\left(1-\frac{\ln \left(P_{A / B, t \mid t_{0}}\right)}{\sigma_{A-B, t}^{2} \tau_{t}}\right)-\Phi\left(d_{1, t}\right) \phi\left(d_{2, t}\right)\left(1+\frac{\ln \left(P_{A / B, t \mid t_{0}}\right)}{\sigma_{A-B, t}^{2} \tau_{t}}\right)\right] .
\end{aligned}
$$

\section{References}

Amenc, N., Malaise, P., \& Martellini, L. (2004). Revisiting core-satellite investing-A dynamic model of relative risk management. Journal of Portfolio Management, 31(1), $64-75$.

Ardia, D., Bolliger, G., Boudt, K., \& Gagnon-Fleury, J.-P. (2017). The impact of covariance misspecification in risk-based portfolios. Annals of Operations Research, 254(1-2), $1-16$.

Ardia, D., Boudt, K., \& Wauters, M. (2016). Smart beta and CPPI performance. Finance,
37(3), 31-65. http://www.en.affi.asso.fr/151-finance-journal.htm.

Black, F., \& Perold, A. (1992). Theory of constant proportion portfolio insurance. Journal of Economic Dynamics and Control, 16(3-4), 403- 426.

Black, F., \& Scholes, M. (1973). The pricing of options and corporate liabilities. Journal of Political Economy, 81(3), 637-654.

Boudt, K., Darras, J., Nguyen, G. H., \& Peeters, B. (2016). Smart beta equity investing through calm and storm. Risk-based and factor investing (pp. 195-225). Elsevier.

Cesari, R., \& Cremonini, D. (2003). Benchmarking, portfolio insurance and technical analysis: a Monte Carlo comparison of dynamic strategies of asset allocation. Journal of Economic Dynamics and Control, 27(6), 987-10 11. 
Jegadeesh, N., \& Titman, S. (1993). Returns to buying winners and selling losers: Implications for stock market efficiency. Journal of Finance, 48(1), 65-91.

Kaminski, K. M., \& Lo, A. W. (2014). When do stop-loss rules stop losses? Journal of Financial Markets, 18, 234-254.

Kolm, P. N., Tütüncü, R., \& Fabozzi, F. J. (2014). 60 years of portfolio optimization: Practical challenges and current trends. European Journal of Operational Research, 234(2), 356- 371

Kula, G., Raab, M., \& Stahn, S. (2017). Beyond smart beta: Index investment strategies for active portfolio management. John Wiley \& Sons.

Margrabe, W. (1978). The value of an option to exchange one asset for another. Journal of Finance, 33(1), 177-186.
Perold, A. F., \& Sharpe, W. F. (1995). Dynamic strategies for asset allocation. Financial Analysts Journal, 51(1), 149-160.

Rubinstein, M., \& Leland, H. E. (1981). Replicating options with positions in stock and cash. Financial Analysts Journal, 37(4), 63-72.

Stoyanov, S. V., Rachev, S. T., \& Fabozzi, F. J. (2013). Sensitivity of portfolio var and cvar to portfolio return characteristics. Annals of Operations Research, 205(1), 169-187. Sullivan, R., Timmermann, A., \& White, H. (1999). Data-snooping, technical trading rule performance, and the bootstrap. Journal of Finance, 54(5), 1647-1 691.

Welch, S. (2008). The hitchhiker's guide to core/satellite investing. The Journal of Wealth Management, 11(3), 97. 